Research Article

\title{
Common Fixed-Point Results in Ordered Left (Right) Quasi- $b$-Metric Spaces and Applications
}

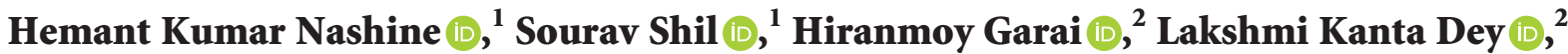 \\ and Vahid Parvaneh (D) $^{3}$
}

\author{
${ }^{1}$ Department of Mathematics, School of Advanced Sciences, Vellore Institute of Technology, Vellore 632014, TN, India \\ ${ }^{2}$ Department of Mathematics, National Institute of Technology, Durgapur, India \\ ${ }^{3}$ Department of Mathematics, Gilan-E-Gharb Branch, Islamic Azad University, Gilan-E-Gharb, Iran
}

Correspondence should be addressed to Vahid Parvaneh; zam.dalahoo@gmail.com

Received 3 September 2020; Revised 6 October 2020; Accepted 19 October 2020; Published 2 December 2020

Academic Editor: Xiaolong Qin

\begin{abstract}
Copyright (C) 2020 Hemant Kumar Nashine et al. This is an open access article distributed under the Creative Commons Attribution License, which permits unrestricted use, distribution, and reproduction in any medium, provided the original work is properly cited.
\end{abstract}

\begin{abstract}
We use the notions of left- and right-complete quasi- $b$-metric spaces and partial ordered sets to obtain a couple of common fixedpoint results for strictly weakly isotone increasing mappings and relatively weakly increasing mappings, which satisfy a pair of almost generalized contractive conditions. To illustrate our results, throughout the paper, we give several relevant examples. Further, we use our results to establish sufficient conditions for existence and uniqueness of solution of a system of nonlinear matrix equations and a pair of fractional differential equations. Finally, we provide a nontrivial example to validate the sufficient conditions for nonlinear matrix equations with numerical approximations.
\end{abstract}

\section{Introduction and Preliminaries}

We denote by $\mathbb{R}$ the set of real numbers; $\mathbb{R}_{+}=[0,+\infty)$; we denote by $\mathbb{N}$ the set of natural numbers and $\mathbb{N}^{*}=\mathbb{N} \cup\{0\}$. Also, for the mappings $\mathscr{T}, \mathcal{S}, \mathscr{R}: \Xi \longrightarrow \Xi$, we denote by CFP $(\mathscr{T}, \mathcal{S})$ and CFP $(\mathscr{T}, \mathcal{S}, \mathscr{R})$ the set of all common fixed points of $\mathscr{T}, \mathcal{S}$ and $\mathscr{T}, \mathcal{S}, \mathscr{R}$, respectively.

The metric fixed-point theory has been extended in many directions by many renowned mathematicians. One important direction of such ones is to revise the underlying metric space to some other spaces by making suitable changes obtained by Czerwik. He introduced the notion of $b$-metric spaces (see [1]), which is further extended as quasi$b$-metric spaces by Shah and Hussain [2].

Definition 1 (see [2]). Let $\Xi(\neq \varnothing)$ be a set and let $b \geq 1$ be a given real number. A function $d_{b}: \Xi^{2} \longrightarrow \mathbb{R}$ is a quasi$b$-metric on $\Xi$ if, for all $\zeta, \xi, \varsigma \in \Xi$,

$$
\begin{aligned}
& \text { (M1) } d_{b}(\zeta, \xi) \geq 0 \\
& \text { (M2) } d_{b}(\zeta, \xi)=0 \Leftrightarrow \zeta=\xi
\end{aligned}
$$

$$
\text { (M3) } d_{b}(\zeta, \varsigma) \leq b\left[d_{b}(\zeta, \xi)+d_{b}(\xi, \varsigma)\right]
$$

The pair $\left(\Xi, d_{b}\right)$ is then termed as a quasi-b-metric space with constant $b$.

It is to be noted that every metric space is quasi-metric space, and quasi-metric space is a quasi- $b$-metric space but the converses need not be true. The above space is further extended with the introduction of right and left quasi$b$-metric spaces (in the line of [3]).

Definition 2 (see [4]). Let $\left(\Xi, d_{b}\right)$ be a quasi- $b$-metric space and let $\left\{\vartheta_{n}\right\}$ be a sequence in $\Xi$. Then $\left\{\vartheta_{n}\right\}$ is said to be

(i) left-Cauchy if, for every $\delta>0$, we get $N=N(\delta) \in \mathbb{N}$ such that $d_{b}\left(\vartheta_{r}, \vartheta_{s}\right)<\delta$ for all $r>s>N$

(ii) right-Cauchy if, for every $\delta>0$, we get $N=N(\delta) \in \mathbb{N}$ such that $d_{b}\left(\vartheta_{r}, \vartheta_{s}\right)<\delta$ for all $s>r>N$

Definition 3 (see [4]). Let $\left(\Xi, d_{b}\right)$ be a quasi- $b$-metric space. Then $\left(\Xi, d_{b}\right)$ is called 
(i) left-complete if every left-Cauchy sequence in $\Xi$ is convergent

(ii) right-complete if every right-Cauchy sequence in $\Xi$ is convergent

On the other hand, an extension of fixed-point results for various types of contractions in metric spaces is secured by adding an (partial) ordering structure on the underlying structure $(\Xi, d)$. Some early results in this direction were established by Turinici in $[5,6]$; one may note that their starting points were "amorphous" contributions in the area due to Matkowski [7, 8]. These types of results have been reinvestigated by Ran and Reurings [9] and also by Nieto and Ródríguez-López [10, 11]. In 2019, Gu and Shatanawi [12] obtained some common coupled fixed-point results in partial metric spaces and some recent results of Latif et al. [13] and Malhotra et at. [14] are also important. In [15], Nashine et al. used the concept of $\mathscr{T}$-weakly isotone increasing mappings to extend Cirić's [16] result in ordered metric spaces. The main importance of their results is that they obtained their results without considering any kind of commutativity condition. After all such generalizations and extensions, Nashine and Altun [17] introduced a new notion of increasing mapping, which they designated as $\mathscr{T}$-strictly weakly isotone increasing mapping, and then they obtained some results by considering this new type of increasing mappings. After this, in [18], Nashine and Samet introduced relatively weakly increasing mappings and proved some fixed-point results in ordered metric spaces and applied their results to integral equations. In this sequel, we like to recall some useful definitions in the context of a partially ordered set $(\Xi, \prec)$.

Definition 4 (see [17-20]). Let $(\Xi, \prec)$ be a partially ordered set and let $\mathcal{S}, \mathscr{T}, \mathscr{R}: \Xi \longrightarrow \Xi$ be three mappings. Then,

(1) $\mathscr{T}$ is called dominating if $\zeta \prec \mathscr{T} \zeta$ for each $\zeta \in \Xi$.

(2) the pair $(\mathcal{S}, \mathscr{T})$ is called weakly increasing if $\mathcal{S} \zeta \prec \mathscr{T} \mathcal{S} \zeta$ and $\mathscr{T} \zeta \prec \mathcal{S} \mathscr{T} \zeta$ for each $\zeta \in \Xi$.

(3) $\mathcal{S}$ is called $\mathscr{T}$-weakly isotone increasing if, for each $\zeta \in \Xi$, we have $\mathcal{S} \zeta \prec \mathscr{T} \mathcal{S} \zeta \prec \mathcal{S} \mathscr{T} \mathcal{S} \zeta$.

(4) the mapping $\mathcal{S}$ is said to be $\mathscr{T}$-strictly weakly isotone increasing if, for $\zeta \in \Xi$ satisfying $\zeta \prec \delta \zeta$, we have $\mathcal{S} \zeta \prec \mathscr{T} \mathcal{S} \zeta \prec \mathcal{S} \mathscr{T} \mathcal{S} \zeta$.

(5) $\mathscr{T}$ and $\mathcal{S}$ are said to be weakly increasing with respect to $\mathscr{R}$ if $\mathscr{T} \Xi \subseteq \mathscr{R} \Xi$ and $\mathcal{S} \Xi \subseteq \mathscr{R} \Xi$ and, for each $\zeta \in \Xi$, we have

$$
\begin{aligned}
\mathscr{T} \zeta & \prec \mathcal{S} \xi, & \text { for all } \xi \in \mathscr{R}^{-1}(\mathscr{T} \zeta), \\
\mathcal{S} \zeta & \prec \mathscr{T} \xi, & \text { for all } \xi \in \mathscr{R}^{-1}(\mathcal{S} \zeta) .
\end{aligned}
$$

Let $\left(\Xi, d_{b}\right)$ be a $b$-metric space. Then two mappings $\mathscr{T}, \mathcal{S}: \Xi \longrightarrow \Xi$ are said to be compatible if $\lim _{n \longrightarrow \infty} d_{b}\left(\mathscr{T} \mathcal{S} \varsigma_{n}, \mathcal{S} \mathscr{T} \varsigma_{n}\right)=0$, for each sequence $\left\{\varsigma_{n}\right\}$ in $\Xi$ with $\lim _{n \longrightarrow \infty} d_{b}\left(\mathscr{T} \varsigma_{n}, \mu\right)=0$ and $\lim _{n \longrightarrow \infty} d_{b}\left(\mathcal{S} \varsigma_{n}, \mu\right)=0$ for some $\mu \in \Xi$. If $\left(\Xi, d_{b}\right)$ is a quasi-b-metric space and $(\Xi, \prec)$ is a partially ordered set, then the triplet $\left(\Xi, d_{b}, \prec\right)$ is called an ordered quasi- $b$-metric space. The space $\left(\Xi, d_{b}, \prec\right)$ is called regular if whenever $\left\{\varsigma_{n}\right\}$ is a nondecreasing sequence in $\Xi$ with respect to $\prec$ and $\varsigma_{n} \longrightarrow \varsigma \in \Xi$ as $n \longrightarrow \infty$, then $\varsigma_{n} \prec \varsigma$ holds.

In the literature of fixed point, one may note that, to find common fixed point of two or more mappings in the setting of different abstract spaces, more specifically in left- and right-complete quasi- $b$-metric spaces, commutativity condition of the mappings plays crucial roles. So it is a challenging work to obtain common fixed point of two or more mappings in such spaces without considering the commutativity condition. One of the main motivations of the paper is to resolve this issue. To proceed with this, we utilize the approaches of Nashine and Altun [17] and Nashine and Samet [18] to obtain some common fixed-point results in the setting of ordered left-complete and right-complete quasi$b$-metric spaces. Firstly, we establish some common fixedpoint theorems for a pair of mappings using the $\mathscr{T}$-strictly weakly isotone increasing condition and without using any kind of commutativity condition in ordered left-complete quasi- $b$-metric spaces. Secondly, we obtain a common fixedpoint result for a triplet of mappings satisfying relatively weakly increasing condition and almost generalized contractive conditions in ordered right-complete quasi- $b$-metric spaces.

Another important motivation of this paper is to show how we can apply our obtained results in at least two different applicable areas. These are connected to get solutions of a pair of nonlinear matrix equations and also a pair of fractional differential equations. Further, we provide some nontrivial examples to illustrate our obtained results. Finally, our attempts give extensions of the works discussed in $[2,3,9-11,15,18,21]$ and other related results in the sense of generalized contractive conditions and generalized weakly increasing mappings in the crucial setting with new applications to the functional equations.

\section{Results for Pair of Mappings}

In this section, at first, we prove a common fixed-point result of a pair of mappings involving $\mathscr{T}$-strictly weakly isotone increasing condition. Before this, we state the following important lemma regarding the left- (right-) Cauchyness of a sequence in quasi-b-metric context.

Lemma 1. Let $\left(\Xi, d_{b}\right)$ be a quasi-b-metric space and let $\left\{x_{n}\right\}$ be a sequence in $\Xi$. Then, we have the following:

(1) If there exists $r \in[0,1)$ satisfying

$$
d_{b}\left(x_{n+2}, x_{n+1}\right) \leq r d_{b}\left(x_{n+1}, x_{n}\right), \quad \text { for all } n \in \mathbb{N},
$$

$$
\text { then }\left\{x_{n}\right\} \text { is a left-Cauchy sequence. }
$$

(2) If there exists $r \in[0,1)$ satisfying

$$
d_{b}\left(x_{n+1}, x_{n+2}\right) \leq r d_{b}\left(x_{n}, x_{n+1}\right), \quad \text { for all } n \in \mathbb{N},
$$

then $\left\{x_{n}\right\}$ is a right-Cauchy sequence. 
Proof. The proof of this lemma can be done in the line of ([22], p. 3, Lemma [23]).
Theorem 1. Let $\left(\Xi, d_{b}, \prec\right)$ be an ordered left-complete quasi-b-metric space with constant $b \geq 1$. Suppose that $\mathscr{T}, \mathcal{S}: \Xi \longrightarrow \Xi$ are two mappings such that

$$
\begin{aligned}
& d_{b}(\mathscr{T} \nu, \mathcal{S} \vartheta) \leq \alpha \Lambda(\nu, \vartheta)+L \min \left\{d_{b}(\nu, \mathscr{T} v), d_{b}(\vartheta, \mathcal{S} \vartheta), d_{b}(\nu, \mathcal{S} \vartheta), d_{b}(\vartheta, \mathscr{T} v)\right\} \\
& d_{b}(\mathcal{S} \nu, \mathscr{T} \vartheta) \leq \alpha \Upsilon(\nu, \vartheta)+L \min \left\{d_{b}(\nu, \mathcal{S} v), d_{b}(\vartheta, \mathscr{T} \vartheta), d_{b}(\nu, \mathscr{T} \vartheta), d_{b}(\vartheta, \mathcal{S} v)\right\}
\end{aligned}
$$

for all comparable $\nu, \vartheta \in \Xi$, where $\alpha \in[0,1), L \geq 0$, and

$$
\begin{aligned}
& \Lambda(\nu, \vartheta)=\max \left\{d_{b}(\nu, \vartheta), d_{b}(\mathscr{T} v, v), \frac{d_{b}(\mathcal{S} \vartheta, \vartheta)+d_{b}(\mathscr{T} v, \nu)}{2}, \frac{d_{b}(\mathscr{T} v, \vartheta)+d_{b}(\mathcal{S} \vartheta, \nu)}{2 b}\right\} \\
& \Upsilon(\nu, \vartheta)=\max \left\{d_{b}(\nu, \vartheta), d_{b}(\mathcal{S} \nu, \nu), \frac{d_{b}(\mathscr{T} \vartheta, \vartheta)+d_{b}(\mathcal{S} \nu, \nu)}{2}, \frac{d_{b}(\mathcal{S} v, \vartheta)+d_{b}(\mathscr{T} \vartheta, \nu)}{2 b},\right\} .
\end{aligned}
$$

In addition, let $\mathcal{S}$ be $\mathscr{T}$-strictly weakly isotone increasing, there exists an $\nu_{0} \in \Xi$ such that $\nu_{0} \prec \mathcal{S} \nu_{0}$, and one of $\mathcal{S}$ and $\mathscr{T}$ is continuous. Then $\operatorname{CFP}(\mathscr{T}, \mathcal{S}) \neq \varnothing$.

Proof. Let $\nu_{0}$ be such that $\nu_{0} \prec \delta \nu_{0}$ and construct a sequence $\left\{\nu_{n}\right\}$ in $\Xi$ satisfying

$v_{2 n+1}=\mathcal{S} v_{2 n}$ and $v_{2 n+2}=\mathscr{T} v_{2 n+1}$ for $n \in\{0,1, \ldots\}$.

As $\mathcal{S}$ is $\mathscr{T}$-weakly isotone increasing,

$$
\begin{aligned}
& v_{1}=\mathcal{S} v_{0} \prec \mathscr{T} \mathcal{S} v_{0}=\mathscr{T} v_{1}=v_{2} \prec \mathcal{S} \mathscr{T} \mathcal{S} v_{0}=\mathcal{S} \mathscr{T} v_{1}=\mathcal{S} v_{2}=v_{3}, \\
& v_{3}=\mathcal{S} v_{2} \prec \mathscr{T} \mathcal{S} v_{2}=\mathscr{T} v_{3}=v_{4} \prec \mathcal{S} \mathscr{T} \mathcal{S} v_{2}=\mathcal{S} \mathscr{T} v_{3}=\mathcal{S} v_{4}=v_{5},
\end{aligned}
$$

and, proceeding with this argument, we get

$$
v_{1} \prec v_{2} \prec \cdots \prec v_{n} \prec v_{n+1} \prec \ldots
$$

Let $\delta_{n}=d_{b}\left(\nu_{n+1}, v_{n}\right)$. Then, for all $n \in \mathbb{N}$, we show that

$$
\delta_{n}<\alpha \delta_{n-1} .
$$

From (10), we have that $v_{n} \prec v_{n+1}$ for all $n \in \mathbb{N}$. Then, from (4), with $\nu=\nu_{2 n+1}$ and $\vartheta=\nu_{2 n}$, we get

$$
\begin{aligned}
d_{b}\left(v_{2 n+2}, v_{2 n+1}\right)= & d_{b}\left(\mathscr{T} v_{2 n+1}, \mathcal{S} v_{2 n}\right), \\
\leq & \alpha \Lambda\left(v_{2 n+1}, v_{2 n}\right), \\
& +L \min \left\{\begin{array}{l}
d_{b}\left(v_{2 n+1}, \mathscr{T} v_{2 n+1}\right), d_{b}\left(v_{2 n}, \mathcal{S} v_{2 n}\right), \\
d_{b}\left(v_{2 n+1}, \mathcal{S} v_{2 n}\right), d_{b}\left(v_{2 n}, \mathscr{T} v_{2 n+1}\right),
\end{array}\right\} \\
\Longrightarrow & d_{b}\left(v_{2 n+2}, v_{2 n+1}\right) \leq \alpha \Lambda\left(v_{2 n+1}, v_{2 n}\right) .
\end{aligned}
$$

By (8), we have

$$
\begin{aligned}
& \Lambda\left(v_{2 n+1}, v_{2 n}\right) \\
& \quad=\max \left\{d_{b}\left(v_{2 n+1}, v_{2 n}\right), d_{b}\left(\mathscr{T} v_{2 n+1}, v_{2 n+1}\right), \frac{d_{b}\left(\mathcal{S} v_{2 n}, v_{2 n}\right)+d_{b}\left(\mathscr{T} v_{2 n+1}, v_{2 n+1}\right)}{2}, \frac{d_{b}\left(\mathscr{T} v_{2 n+1}, v_{2 n}\right)+d_{b}\left(\mathcal{S} v_{2 n}, v_{2 n+1}\right)}{2 b}\right\} \\
& \quad=\max \left\{d_{b}\left(v_{2 n+1}, v_{2 n}\right), d_{b}\left(v_{2 n+2}, v_{2 n+1}\right), \frac{d_{b}\left(v_{2 n+1}, v_{2 n}\right)+d_{b}\left(v_{2 n+2}, v_{2 n+1}\right)}{2}, \frac{d_{b}\left(v_{2 n+2}, v_{2 n}\right)+d_{b}\left(v_{2 n+1}, v_{2 n+1}\right)}{2 b}\right\} \\
& \quad=\max \left\{d_{b}\left(v_{2 n+1}, v_{2 n}\right), d_{b}\left(v_{2 n+2}, v_{2 n+1}\right), \frac{d_{b}\left(v_{2 n+1}, v_{2 n}\right)+d_{b}\left(v_{2 n+2}, v_{2 n+1}\right)}{2}, \frac{d_{b}\left(v_{2 n+2}, v_{2 n}\right)}{2 b}\right\}
\end{aligned}
$$


(i) If $\Lambda\left(v_{2 n+1}, v_{2 n}\right)=d_{b}\left(v_{2 n+2}, v_{2 n+1}\right)$, by (12), we have $d_{b}\left(\nu_{2 n+2}, v_{2 n+1}\right) \leq \alpha\left(d_{b}\left(\nu_{2 n+2}, v_{2 n+1}\right)\right)<d_{b}\left(\nu_{2 n+2}, v_{2 n+1}\right)$,

a contradiction.

(ii) If $\Lambda\left(v_{2 n+1}, v_{2 n}\right)=\left(d_{b}\left(v_{2 n+2}, v_{2 n}\right) / 2 b\right)$, we have

$$
\begin{aligned}
d_{b}\left(v_{2 n+2}, v_{2 n+1}\right) & \leq \alpha \frac{d_{b}\left(v_{2 n+2}, v_{2 n}\right)}{2 b} \\
& \leq \frac{\alpha}{2}\left\{d_{b}\left(v_{2 n+2}, v_{2 n+1}\right)+d_{b}\left(v_{2 n+1}, v_{2 n}\right)\right\} \\
& \Longrightarrow d_{b}\left(v_{2 n+2}, v_{2 n+1}\right) \\
& \leq \frac{\alpha}{2-\alpha} d_{b}\left(v_{2 n+1}, v_{2 n}\right) \Longrightarrow d_{b}\left(v_{2 n+2}, v_{2 n+1}\right) \\
& \leq \alpha d_{b}\left(v_{2 n+1}, v_{2 n}\right) \Longrightarrow \delta_{2 n+1}, \\
& \leq \alpha \delta_{2 n}
\end{aligned}
$$

(iii) If $\Lambda\left(v_{2 n+1}, v_{2 n}\right)=d_{b}\left(v_{2 n+1}, v_{2 n}\right)$, we have

$d_{b}\left(\nu_{2 n+2}, \nu_{2 n+1}\right) \leq \alpha d_{b}\left(\nu_{2 n+1}, \nu_{2 n}\right) \Longrightarrow \delta_{2 n+1} \leq \alpha \delta_{2 n}$.

(iv) If $\Lambda\left(v_{2 n+1}, v_{2 n}\right)=\left(\left(d_{b}\left(\nu_{2 n+1}, v_{2 n}\right)+d_{b}\left(\nu_{2 n+2}, v_{2 n+1}\right)\right) / 2\right)$, we have

$$
\begin{aligned}
d_{b}\left(v_{2 n+2}, v_{2 n+1}\right) & \leq \alpha \frac{d_{b}\left(v_{2 n+1}, v_{2 n}\right)+d_{b}\left(v_{2 n+2}, v_{2 n+1}\right)}{2} \\
& \Longrightarrow d_{b}\left(v_{2 n+2}, v_{2 n+1}\right) \\
& \leq \frac{\alpha}{2-\alpha} d_{b}\left(v_{2 n+1}, v_{2 n}\right) \Longrightarrow d_{b}\left(v_{2 n+2}, v_{2 n+1}\right) \\
& \leq \alpha d_{b}\left(v_{2 n+1}, v_{2 n}\right) \Longrightarrow \delta_{2 n+1} \\
& \leq \alpha \delta_{2 n} .
\end{aligned}
$$

Consequently, $\delta_{2 n+1}<\alpha \delta_{2 n}$ for all $n \in \mathbb{N}$. Similarly, using (5) with (7), we can show that $\delta_{2 n}<\alpha \delta_{2 n-1}$. Therefore, (11) holds for all $n \in \mathbb{N}$ and so, from Lemma 1 , we can conclude that $\left\{v_{n}\right\}$ is a left-Cauchy sequence.

From the left-completeness of $\Xi$, there exists $\varrho \in \Xi$ such that $v_{n} \longrightarrow \varrho$ as $n \longrightarrow+\infty$. Clearly, if $\mathcal{S}$ or $\mathscr{T}$ is continuous, then $\varrho=\delta \varrho$ or $\varrho=\mathscr{T} \varrho$. Thus, $\operatorname{CFP}(\mathscr{T}, \mathcal{S}) \neq \varnothing$.By the next result, we show that the continuity of $\mathcal{S}$ or $\mathscr{T}$ in the previous theorem can be replaced by some other conditions.

Theorem 2. If one replaces the continuity in Theorem 1 by regularity of $\Xi$, then conclusion of Theorem 1 is valid provided $\alpha b<1$.

Proof. Following the lines of proof of Theorem 1, we have that there exists $\varrho \in \Xi$ such that

$$
\lim _{n \longrightarrow+\infty} v_{n}=\lim _{n \longrightarrow+\infty} \mathcal{S} v_{2 n}=\lim _{n \longrightarrow+\infty} \mathscr{T} v_{2 n+1}=\varrho
$$

Using (5) for $\vartheta=v_{2 n+1}$ and $\nu=\varrho$, we have

$$
\begin{aligned}
& d_{b}(\mathcal{S} \varrho, \varrho) \\
& \quad \leq b\left[d_{b}\left(\mathcal{S} \varrho, \mathscr{T} v_{2 n+1}\right)+d_{b}\left(\mathscr{T} v_{2 n+1}, \varrho\right)\right], \\
& \quad \leq \alpha b \max \left\{d_{b}\left(\varrho, v_{2 n+1}\right), d_{b}(\mathcal{S} \varrho, \varrho), \frac{d_{b}\left(\mathscr{T} v_{2 n+1}, v_{2 n+1}\right)+d_{b}(\mathcal{S} \varrho, \varrho)}{2}, \frac{d_{b}\left(\mathcal{S} \varrho, v_{2 n+1}\right)+d_{b}\left(\mathscr{T} v_{2 n+1}, \varrho\right)}{2 b}\right\}, \\
& \quad+L b \min \left\{d_{b}(\varrho, \mathcal{S} \varrho), d_{b}\left(v_{2 n+1}, \mathscr{T} v_{2 n+1}\right), d_{b}\left(\varrho, \mathscr{T} v_{2 n+1}\right), d_{b}\left(v_{2 n+1}, \mathcal{S} \varrho\right)\right\}, \\
& \quad+b d_{b}\left(\mathscr{T} v_{2 n+1}, \varrho\right) .
\end{aligned}
$$


Letting $n \longrightarrow \infty$ in the above equation, we get

$$
d_{b}(\mathcal{S} \varrho, \varrho) \leq \max \alpha b\left\{d_{b}(\mathcal{S} \varrho, \varrho), \frac{1}{2} d_{b}(\mathcal{S} \varrho, \varrho), \frac{1}{2 b} d_{b}(\mathcal{S} \varrho, \varrho)\right\}=\alpha b d_{b}(\mathcal{S} \varrho, \varrho) .
$$

Hence $d_{b}(\mathcal{S} \varrho, \varrho)=0$ and so $\mathcal{S} \varrho=\varrho$. Analogously, using (4) for $v=\varrho$ and $\vartheta=v_{2 n}$, we get $\mathscr{T} \varrho=\varrho$. It follows that $\varrho \in \operatorname{CFP}(\mathscr{T}, \mathcal{S})$; that is, $\operatorname{CFP}(\mathscr{T}, \mathcal{S}) \neq \varnothing$.

Next, we characterize the common fixed-points set $\operatorname{CFP}(\mathscr{T}, \mathcal{S})$ in the following theorem.

$$
\begin{aligned}
d_{b}(\sigma, \varrho)= & d_{b}(\mathscr{T} \sigma, \mathcal{S} \varrho) \leq \alpha \Lambda(\sigma, \varrho), \\
& +L \min \left\{d_{b}(\sigma, \mathscr{T} \sigma), d_{b}(\varrho, \mathcal{S} \varrho), d_{b}(\sigma, \mathcal{S} \varrho), d_{b}(\varrho, \mathscr{T} \sigma)\right\}, \\
= & \alpha \max \left\{d_{b}(\sigma, \varrho), d_{b}(\mathscr{T} \sigma, \sigma), \frac{d_{b}(\mathcal{S} \varrho, \varrho)+d_{b}(\mathscr{T} \sigma, \varrho)}{2}, \frac{d_{b}(\mathscr{T} \sigma, \varrho)+d_{b}(\mathcal{S} \varrho, \sigma)}{2 b}\right\}, \\
= & \alpha \max \left\{d_{b}(\sigma, \varrho), \frac{d_{b}(\sigma, \varrho)+d_{b}(\varrho, \sigma)}{2 b}\right\}, \\
= & \frac{\alpha}{2 b}\left\{d_{b}(\sigma, \varrho)+d_{b}(\varrho, \sigma)\right\},
\end{aligned}
$$

that is,

$$
d_{b}(\sigma, \varrho) \leq \frac{\alpha}{2 b}\left\{d_{b}(\sigma, \varrho)+d_{b}(\varrho, \sigma)\right\}
$$

Again, using (5) for $v=u$ and $\vartheta=\sigma$ and by calculation we get

$$
d_{b}(\varrho, \sigma) \leq \frac{\alpha}{2 b}\left\{d_{b}(\varrho, \sigma)+d_{b}(\sigma, \varrho)\right\} .
$$

Adding (22) and (23), we get
Theorem 3. Let all the conditions of Theorems 1 and 2 hold. Then $\operatorname{CFP}(\mathscr{T}, \mathcal{S})$ is totally ordered if and only if $\operatorname{CFP}(\mathscr{T}, \mathcal{S})$ contains exactly one element.

Proof. First, we assume that $\operatorname{CFP}(\mathscr{T}, \mathcal{S})$ is totally ordered. Let $\varrho, \sigma \in \operatorname{CFP}(\mathscr{T}, \mathcal{S})$ with $\varrho \neq \sigma$. Consider (4) for $v=\sigma$ and $\vartheta=\varrho$, and we get

$$
d_{b}(\mathscr{T} \nu, \mathscr{T} \vartheta) \leq \alpha \Lambda^{\prime}(\nu, \vartheta)+L \min \left\{d_{b}(\nu, \mathscr{T} v), d_{b}(\vartheta, \mathscr{T} \vartheta), d_{b}(\nu, \mathscr{T} \vartheta), d_{b}(\vartheta, \mathscr{T} \nu)\right\}
$$

for all comparable $\nu, \vartheta \in \Xi$, where $L \geq 0, \alpha \in[0,1)$, and

$$
\Lambda^{\prime}(\nu, \vartheta)=\max \left\{d_{b}(\nu, \vartheta), d_{b}(\mathscr{T} \nu, \nu), \frac{d_{b}(\mathscr{T} \vartheta, \vartheta)+d_{b}(\mathscr{T} \nu, \nu)}{2}, \frac{d_{b}(\mathscr{T} \nu, \vartheta)+d_{b}(\mathscr{T} \vartheta, \nu)}{2 b}\right\}
$$

Also suppose that $\mathscr{T} \nu \prec \mathscr{T}(\mathscr{T} \nu)$ for all $\nu \in \Xi$ with $\nu \prec \mathscr{T} v$. If there exists an element $\nu_{0} \in \Xi$ such that $\nu_{0} \prec \mathscr{T} \nu_{0}$ and either $\mathscr{T}$ is continuous at $\nu_{0}$ or $\Xi$ is regular, then $\mathscr{T}$ has a fixed point. Moreover, fix $(\mathscr{T})$ is totally ordered if and only if it is a singleton.
Next, we come up with the following example, which illustrates Theorem 1.

Example 1. Let $\Xi=[0,5]$ and define $d_{b}: \Xi \times \Xi \longrightarrow \mathbb{R}$ by 


$$
d_{b}(\nu, \vartheta)=\left\{\begin{array}{l}
0, \quad \text { if } v=\vartheta \\
\nu-\vartheta, \quad \text { if } \nu>\vartheta ; \\
2(\vartheta-v), \quad \text { if } \nu<\vartheta .
\end{array}\right.
$$

We define a relation “ $\prec$ ” on $\Xi$ by $\nu \prec \vartheta$ if and only if either $\nu, y \in[0,3]$ and $\nu \geq \vartheta$ or $\nu, \vartheta \in(3,5]$ and $\nu=\vartheta$. Then $\left(\Xi, d_{b}, \prec\right)$ is an ordered left-complete quasi-b-metric space with constant $b=2$. Next, we define two mappings $\mathscr{T}, \mathcal{S}: \Xi \longrightarrow \Xi$ by

$$
\begin{aligned}
& \mathscr{T} v= \begin{cases}\frac{v}{8}, & \text { if } v \in[0,3] ; \\
\frac{3}{8}, & \text { if } v \in(3,5],\end{cases} \\
& \delta \mathcal{S}= \begin{cases}\frac{v}{7}, & \text { if } v \in[0,3] ; \\
2, & \text { if } v \in(3,5] .\end{cases}
\end{aligned}
$$

For $v \in[0,3]$ with $v \prec \delta \mathcal{S}$, we have $\delta v=(x / 7)$, $T \mathcal{S} v=(\nu / 56)$, and $\mathcal{S} \mathscr{T} \mathcal{S} v=(\nu / 392)$. So $\mathcal{S} v>\mathscr{T} \mathcal{S} v>$ $\mathcal{S} \mathscr{T} \mathcal{S} v$ and hence $\mathcal{S} v>\mathscr{T} \mathcal{S} v>\mathcal{S} \mathscr{T} \mathcal{S} v$. Again, for $v \in(3,5]$ and $v \prec \mathcal{S} v$, we have $\mathcal{S} v=2, \quad \mathscr{T} \mathcal{S} v=(1 / 4)$, and

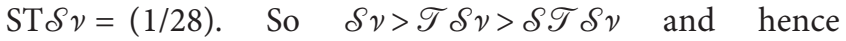
$\mathcal{S} v \prec \mathscr{T} \mathcal{S} v \prec \mathcal{S} \mathscr{T} \mathcal{S} v$. Thus, $\mathcal{S}$ is $\mathscr{T}$-strictly weakly isotone increasing. It is clear that $\mathscr{T}$ is continuous. We choose $\alpha=(4 / 10)$.

Now assume that $\nu, \vartheta \in \Xi$ is arbitrary such that $\nu$ and $\vartheta$ are comparable. Then the following cases arise:

Case I: let $v, \vartheta \in[0,3]$ and $\nu \prec \vartheta$; that is, $v \geq \vartheta$. First, we assume that $\nu>\vartheta$. Then

$$
d_{b}(\mathscr{T} \nu, \mathcal{S} \vartheta) \leq \begin{cases}\frac{v}{8}>\frac{\vartheta}{7}, & \text { if } \frac{v}{8}>\frac{\vartheta}{7} ; \\ 2\left(\frac{\vartheta}{8}>\frac{\nu}{7}\right), & \text { if } \frac{v}{8}<\frac{\vartheta}{7},\end{cases}
$$

and $\quad \alpha d_{b}(\nu, \vartheta)=(4 / 10)(\nu-\vartheta)$ and $\alpha d_{b}(\mathscr{T} \nu, \nu)=$ (7/10). Therefore,

$$
d_{b}(\mathscr{T} \nu, \mathcal{\vartheta}) \leq \begin{cases}\alpha d_{b}(\nu, \vartheta), & \text { if } \frac{\nu}{8}>\frac{\vartheta}{7} \\ \alpha d_{b}(\mathscr{T} \nu, \nu), & \text { if } \frac{\nu}{8}<\frac{\vartheta}{7}\end{cases}
$$

Next, we assume that $\nu=\vartheta$. Then $d_{b}(\mathscr{T} \nu, \mathcal{S} \vartheta)=(\nu / 28)$ and so

$$
d_{b}(\mathscr{T} v, \delta \vartheta) \leq \alpha d_{b}(\mathscr{T} \nu, \nu)
$$

Thus,

$$
d_{b}(\mathscr{T} \nu, \mathcal{\vartheta} \vartheta) \leq \alpha \Lambda(\nu, \vartheta)
$$

On the similar arguments, we can get

$$
d_{b}(\mathcal{S} \nu, \mathscr{T} \vartheta) \leq \alpha \Upsilon(\nu, \vartheta)
$$

Case II: let $\nu, \vartheta \in[0,3]$ and $\vartheta \prec v$; that is, $v \leq \vartheta$. Then, we can show in a similar manner that

$$
\begin{gathered}
\left.d_{b}(\mathscr{T} \nu, \mathcal{S} \vartheta) \leq \alpha \Lambda(\nu, \vartheta)\right), \\
d_{b}(\mathcal{S} \nu, \mathscr{T} \vartheta) \leq \alpha \Upsilon(\nu, \vartheta) .
\end{gathered}
$$

Case III: let $\nu, \vartheta \in(3,5]$ and $v=\vartheta$. Then $d_{b}(\mathscr{T} v, \mathcal{S} \vartheta)=$ $13 / 4$ and $d_{b}(\mathcal{S} v, \mathscr{T} \vartheta)=13 / 8$. Also, we have

$$
\begin{aligned}
& \min \left\{d_{b}(\nu, \mathscr{T} v), d_{b}(\vartheta, \mathcal{S} \vartheta), d_{b}(v, \mathcal{S} \vartheta), d_{b}(\vartheta, \mathscr{T} v)\right\}=\min \left\{\left(\nu-\frac{3}{8}\right),(\nu-2)\right\}=(\nu-2) \\
& \min \left\{d_{b}(\nu, \mathcal{S} v), d_{b}(\vartheta, \mathscr{T} \vartheta), d_{b}(\nu, \mathscr{T} \vartheta), d_{b}(\vartheta, \mathcal{S} v)\right\}=(\nu-2)
\end{aligned}
$$

Therefore,

$$
\begin{aligned}
& d_{b}(\mathscr{T} v, \mathcal{S} \vartheta) \leq L \min \left\{d_{b}(\nu, \mathscr{T} v), d_{b}(\vartheta, \mathcal{S} \vartheta), d_{b}(\nu, \mathcal{S} \vartheta), d_{b}(\vartheta, \mathscr{T} v)\right\} \\
& d_{b}(\mathcal{S} \nu, \mathscr{T} \vartheta) \leq L \min \left\{d_{b}(\nu, \mathcal{S} v), d_{b}(\vartheta, \mathscr{T} \vartheta), d_{b}(\nu, \mathscr{T} \vartheta), d_{b}(\vartheta, \mathcal{S} v)\right\}
\end{aligned}
$$

where $L=10$. 
Hence, from all three cases, we have

$$
\begin{aligned}
& d_{b}(\mathscr{T} v, \mathcal{S} \vartheta) \leq \alpha \Lambda(\nu, \vartheta)+L \min \left\{d_{b}(\nu, \mathscr{T} v), d_{b}(\vartheta, \mathcal{S} \vartheta), d_{b}(\nu, \mathcal{S} \vartheta), d_{b}(\vartheta, \mathscr{T} v)\right\} \\
& d_{b}(\mathcal{S} v, \mathscr{T} \vartheta) \leq \alpha \Upsilon(\nu, \vartheta)+L \min \left\{d_{b}(\nu, \mathcal{S} v), d_{b}(\vartheta, \mathscr{T} \vartheta), d_{b}(\nu, \mathscr{T} \vartheta), d_{b}(\vartheta, \mathcal{S} v)\right\}
\end{aligned}
$$

Thus, all the conditions of Theorem 1 are fulfilled and the pair $\mathscr{T}, \mathcal{S}$ has a unique common fixed point (which is $0 \in \operatorname{CFP}(\mathscr{T}, \mathcal{S})$ ).

\section{Results for Three Mappings}

In this section, we obtain a result for three mappings involving weakly increasing condition.
Theorem 4. Let $\left(\Xi, d_{b}, \prec\right)$ be an ordered right-complete quasi-b-metric space with constant $b \geq 1$. Let $\mathscr{T}, \mathcal{S}, \mathscr{R}: \Xi \longrightarrow \Xi$ be three mappings such that $\mathscr{T} \Xi \subseteq \mathscr{R} \Xi$ and $\mathcal{S} \Xi \subseteq \mathscr{R} \Xi$, and

$$
\begin{aligned}
& d_{b}(\mathscr{T} v, \mathcal{S} \vartheta) \leq \alpha \Lambda_{1}(\nu, \vartheta)+L \min \left\{d_{b}(\mathscr{R} v, \mathscr{T} v), d_{b}(\mathscr{R} \vartheta, \mathcal{S} \vartheta), d_{b}(\mathscr{R} v, \mathcal{S} \vartheta), d_{b}(\mathscr{R} \vartheta, \mathscr{T} v),\right\}, \\
& d_{b}(\mathcal{S} v, \mathscr{T} \vartheta) \leq \alpha Y_{1}(\nu, \vartheta)+L \min \left\{d_{b}(\mathscr{R} v, \mathcal{S} v), d_{b}(\mathscr{R} \vartheta, \mathscr{T} \vartheta), d_{b}(\mathscr{R} v, \mathscr{T} \vartheta), d_{b}(\mathscr{R} \vartheta, \mathcal{S} v)\right\},
\end{aligned}
$$

for all comparable $\mathscr{R} v, \mathscr{R} y \in \Xi$, where $\alpha \in[0,1), L \geq 0$, and

$$
\begin{aligned}
& \Lambda_{1}(\nu, \vartheta)=\max \left\{d_{b}(\mathscr{R} v, \mathscr{R} \vartheta), d_{b}(\mathscr{R} v, \mathscr{T} v), \frac{d_{b}(\mathscr{R} \vartheta, \mathcal{S} \vartheta)+d_{b}(\mathscr{R} v, \mathscr{T} v)}{2}, \frac{d_{b}(\mathscr{T} v, \mathscr{R} \vartheta)+d_{b}(\mathscr{R} v, \mathcal{S} \vartheta)}{2 b}\right\}, \\
& Y_{1}(\nu, \vartheta)=\max \left\{d_{b}(\mathscr{R} v, \mathscr{R} \vartheta), d_{b}(\mathscr{R} v, \mathcal{S} v), \frac{d_{b}(\mathscr{R} \vartheta, \mathscr{T} \vartheta)+d_{b}(\mathscr{R} v, \mathcal{S} v)}{2}, \frac{d_{b}(\mathcal{S} v, \mathscr{R} \vartheta)+d_{b}(\mathscr{R} v, \mathscr{T} \vartheta)}{2 b}\right\} .
\end{aligned}
$$

We assume the following hypotheses:

(i) $\mathscr{T}$ and $\mathcal{S}$ are weakly increasing with respect to $\mathscr{R}$

(ii) $\mathscr{T}$ and $\mathcal{S}$ are dominating maps

Assume either of the following:

(a) $\mathcal{S}$ and $\mathscr{R}$ are compatible; $\mathscr{R}$ is continuous at $\nu_{0}$

(b) $\mathscr{T}$ and $\mathscr{R}$ are compatible; $\mathscr{R}$ is continuous at $v_{0}$

Then $\operatorname{CFP}(\mathcal{S}, \mathscr{T}, \mathscr{R}) \neq \varnothing$. In addition, $\operatorname{CFP}(\mathcal{S}, \mathscr{T}, \mathscr{R})$ is totally ordered if and only if $\mathrm{CFP}(\mathcal{S}, \mathscr{T}, \mathscr{R})$ contains exactly one element.

Proof. Start with defining a sequence $\left\{v_{n}\right\}$ in $\Xi$ as

$$
\mathscr{R} v_{2 n+1}=\mathcal{S} v_{2 n} \text { and } \mathscr{R} v_{2 n+2}=\mathscr{T} v_{2 n+1} \text { for } n \in\{0,1, \ldots\} \text {. }
$$

We claim that

$$
\mathscr{R} v_{n} \prec \mathscr{R} v_{n+1}, \quad \forall n \in \mathbb{N}^{*} .
$$

Using hypothesis (i) and (41),

$$
\mathscr{R} v_{1}=\mathcal{S} v_{0} \prec \mathscr{T} \mathcal{\vartheta}, \quad \forall y \in \mathscr{R}^{-1}\left(\mathcal{S} v_{0}\right) .
$$

Since $\mathscr{R} v_{1}=\delta v_{0}, v_{1} \in \mathscr{R}^{-1}\left(\delta v_{0}\right)$, and we get

$$
\mathscr{R} v_{1}=\delta v_{0} \prec \mathscr{T} v_{1}=\mathscr{R} v_{2} \text {. }
$$

Again,

$$
\mathscr{R} v_{2}=\mathscr{T} v_{1} \prec \mathcal{S} \vartheta, \quad \forall y \in \mathscr{R}^{-1}\left(\mathscr{T} v_{1}\right) .
$$

Since $v_{2} \in \mathscr{R}^{-1}\left(\mathscr{T} v_{1}\right)$, we get

$$
\mathscr{R} v_{2}=\mathscr{T} v_{1} \prec \delta v_{2}=\mathscr{R} v_{3} .
$$

Hence, by induction, (42) holds.

Let $\Delta_{n}=d_{b}\left(\mathscr{R} v_{n}, \mathscr{R} v_{n+1}\right)$. Now we claim that, for all $n \in \mathbb{N}$, we have

$$
\Delta_{n} \leq \alpha \Delta_{n-1} .
$$

Using (38) and (42) with $x v=v_{2 n-1}$ and $\vartheta=v_{2 n}$, 


$$
\begin{aligned}
d_{b}\left(\mathscr{R} v_{2 n}, \mathscr{R} v_{2 n+1}\right) & =d_{b}\left(\mathscr{T} v_{2 n-1}, \mathcal{S} v_{2 n}\right) \\
& \leq \alpha \Lambda_{1}\left(v_{2 n-1}, v_{2 n}\right)+L \min \left\{d_{b}\left(\mathscr{R} v_{2 n-1}, \mathscr{T} v_{2 n-1}\right), d_{b}\left(\mathscr{R} v_{2 n}, \mathcal{S} v_{2 n}\right), d_{b}\left(\mathscr{R} v_{2 n-1}, \mathcal{S} v_{2 n}\right), d_{b}\left(\mathscr{R} v_{2 n}, \mathscr{T} v_{2 n-1}\right)\right\} \\
& \Longrightarrow d_{b}\left(\mathscr{R} v_{2 n}, \mathscr{R} v_{2 n+1}\right) \leq \alpha \Lambda_{1}\left(v_{2 n-1}, v_{2 n}\right)
\end{aligned}
$$

where

$$
\begin{aligned}
& \Lambda_{1}\left(v_{2 n-1}, v_{2 n}\right) \\
& =\max \left\{d_{b}\left(\mathscr{R} v_{2 n-1}, \mathscr{R} v_{2 n}\right), d_{b}\left(\mathscr{R} v_{2 n-1}, \mathscr{T} v_{2 n-1}\right), \frac{d_{b}\left(\mathscr{R} v_{2 n}, \mathcal{S} v_{2 n}\right)+d_{b}\left(\mathscr{R} v_{2 n-1}, \mathscr{T} v_{2 n-1}\right)}{2}, \frac{d_{b}\left(\mathscr{T} v_{2 n-1}, \mathscr{R} v_{2 n}\right)+d_{b}\left(\mathscr{R} v_{2 n-1}, \mathcal{S} v_{2 n}\right)}{2 b}\right\} \\
& =\max \left\{d_{b}\left(\mathscr{R} v_{2 n-1}, \mathscr{R} v_{2 n}\right), d_{b}\left(\mathscr{R} v_{2 n-1}, \mathscr{R} v_{2 n}\right), \frac{d_{b}\left(\mathscr{R} v_{2 n}, \mathscr{R} v_{2 n+1}\right)+d_{b}\left(\mathscr{R} v_{2 n-1}, \mathscr{R} v_{2 n}\right)}{2}, \frac{d_{b}\left(\mathscr{R} v_{2 n-1}, \mathscr{R} v_{2 n+1}\right)}{2 b}\right\} .
\end{aligned}
$$

(i) If $\Lambda_{1}\left(v_{2 n+1}, v_{2 n}\right)=d_{b}\left(\mathscr{R} v_{2 n-1}, \mathscr{R} v_{2 n}\right)$, by (48), we have

$$
d_{b}\left(\mathscr{R} v_{2 n}, \mathscr{R} v_{2 n+1}\right) \leq \alpha d_{b}\left(\mathscr{R} v_{2 n-1}, \mathscr{R} v_{2 n}\right)
$$

that is, $\Delta_{2 n} \leq \alpha \Delta_{2 n-1}$.

(ii) If $\Lambda_{1}\left(v_{2 n-1}, v_{2 n}\right)=(1 / 2 b) d_{b}\left(\mathscr{R} v_{2 n-1}, \mathscr{R} v_{2 n+1}\right)$, we get

$$
\begin{aligned}
d_{b}\left(\mathscr{R} v_{2 n}, \mathscr{R} v_{2 n+1}\right) & \leq \frac{\alpha}{2 b} d_{b}\left(\mathscr{R} v_{2 n-1}, \mathscr{R} v_{2 n+1}\right), \\
& \leq \frac{\alpha}{2} d_{b}\left(\mathscr{R} v_{2 n-1}, \mathscr{R} v_{2 n}\right)+\frac{\alpha}{2} d_{b}\left(\mathscr{R} v_{2 n}, \mathscr{R} v_{2 n+1}\right) \\
& \Longrightarrow d_{b}\left(\mathscr{R} v_{2 n}, \mathscr{R} v_{2 n+1}\right), \\
& \leq \frac{\alpha}{2-\alpha} d_{b}\left(\mathscr{R} v_{2 n-1}, \mathscr{R} v_{2 n}\right), \\
& \leq \alpha d_{b}\left(\mathscr{R} v_{2 n-1}, \mathscr{R} v_{2 n}\right) \Longrightarrow \Delta_{2 n}, \\
& \leq \alpha \Delta_{2 n-1} .
\end{aligned}
$$

(iii) If $\quad \Lambda_{1}\left(v_{2 n-1}, v_{2 n}\right)=\left(\left(d_{b} \quad\left(\mathscr{R} v_{2 n}, \mathscr{R} v_{2 n+1}\right)+d_{b}\right.\right.$ $\left.\left.\left(\mathscr{R} v_{2 n-1}, \mathscr{R} v_{2 n}\right)\right) / 2\right)$, we get

$\leq \frac{\alpha}{2} d_{b}\left(\mathscr{R} v_{2 n}, \mathscr{R} v_{2 n+1}\right)+\frac{\alpha}{2} d_{b}\left(\mathscr{R} v_{2 n-1}, \mathscr{R} v_{2 n}\right) \Longrightarrow \Delta_{2 n}$

$\leq \alpha \Delta_{2 n-1}$.

$$
\begin{aligned}
& d_{b}\left(\mathscr{T} v_{2 n+1}, \mathcal{S} \mathscr{R} v_{2 n+2}\right) \\
& \quad \leq \alpha \Lambda_{1}\left(v_{2 n+1}, \mathscr{R} v_{2 n+2}\right)+L \min \left\{d_{b}\left(\mathscr{R} v_{2 n+1}, \mathscr{T} v_{2 n+1}\right), d_{b}\left(\mathscr{R}\left(\mathscr{R} v_{2 n+2}\right), \mathcal{S} \mathscr{R} v_{2 n+2}\right), d_{b}\left(\mathscr{R} v_{2 n+1}, \mathcal{S} \mathscr{R} v_{2 n+2}\right), d_{b}\left(\mathscr{R}\left(\mathscr{R} v_{2 n+2}\right), \mathscr{T} v_{2 n+1}\right)\right\},
\end{aligned}
$$

Thus, in all cases, we have $\Delta_{2 n} \leq \alpha \Delta_{2 n-1}$ for all $n \in \mathbb{N}$. Similarly, using (39), we can show $\Delta_{2 n+1} \leq \alpha \Delta_{2 n}$ for all $n \geq 1$. Therefore, we conclude that (47) holds for all $n \in \mathbb{N}$; that is,

$$
d_{b}\left(\mathscr{R} v_{n}, \mathscr{R} v_{n+1}\right) \leq \alpha d_{b}\left(\mathscr{R} v_{n-1}, \mathscr{R} v_{n}\right), \quad \text { for all } n \in \mathbb{N} \text {. }
$$

Therefore, from Lemma 1 , it follows that $\left\{\mathscr{R} v_{n}\right\}$ is a right-Cauchy sequence. From the right-completeness of $\Xi$, there exists $\varrho \in \Xi$ such that

$$
\mathscr{R} v_{n} \longrightarrow \varrho \text { as } n \longrightarrow \infty \text {. }
$$

We will prove that $\varrho$ is a common fixed point of the three mappings $\mathcal{S}, \mathscr{T}$, and $\mathscr{R}$.

We have

$$
\begin{gathered}
\mathscr{R} v_{2 n+1}=\mathcal{S} v_{2 n} \longrightarrow \varrho, \quad \text { as } n \longrightarrow \infty, \\
\mathscr{R} v_{2 n+2}=\mathscr{T} v_{2 n+1} \longrightarrow \varrho, \quad \text { as } n \longrightarrow \infty .
\end{gathered}
$$

First, suppose that (a) holds. Then

$$
\lim _{n \longrightarrow \infty} \mathcal{S} \mathscr{R} v_{2 n+2}=\lim _{n \longrightarrow \infty} \mathscr{R} \mathcal{S} v_{2 n+2}=\mathscr{R} \varrho .
$$

From (54) and the continuity of $\mathscr{R}$, we have

$$
\mathscr{R}\left(\mathscr{R} v_{n}\right) \longrightarrow \mathscr{R} z, \quad \text { as } n \longrightarrow \infty \text {. }
$$

Also, we have $v_{2 n+1} \prec \mathscr{T} v_{2 n+1}=\mathscr{R} v_{2 n+2}$. Then, from (38), we get 
where

$$
\begin{aligned}
\Lambda_{1}\left(v_{2 n+1}, \mathscr{R} v_{2 n+2}\right)= & \max \left\{d_{b}\left(\mathscr{R} v_{2 n+1}, \mathscr{R}\left(\mathscr{R} v_{2 n+2}\right)\right), d_{b}\left(\mathscr{R} v_{2 n+1}, \mathscr{T} v_{2 n+1}\right),\right. \\
& \left.\frac{d_{b}\left(\mathscr{R}\left(\mathscr{R} v_{2 n+2}\right), \mathcal{S} \mathscr{R} v_{2 n+2}\right)+d_{b}\left(\mathscr{R} v_{2 n+1}, \mathscr{T} v_{2 n+1}\right)}{2}, \frac{d_{b}\left(\mathscr{T} v_{2 n+1}, \mathscr{R}\left(\mathscr{R} v_{2 n+2}\right)\right)+d_{b}\left(\mathscr{R} v_{2 n+1}, \mathcal{S} \mathscr{R} v_{2 n+2}\right)}{2 b}\right\} .
\end{aligned}
$$

Limiting $n \longrightarrow \infty$ in (59) and using (55)-(58), we obtain

$$
\begin{aligned}
d_{b}(\varrho, \mathscr{R} \varrho) & \leq \alpha \max \left\{d_{b}(\varrho, \mathscr{R} \varrho), \frac{d_{b}(\varrho, \mathscr{R} \varrho)+d_{b}(\varrho, \mathscr{R} \varrho)}{2 b}\right\}, \\
& =\alpha d_{b}(\varrho, \mathscr{R} \varrho) \Longrightarrow d_{b}(\varrho, \mathscr{R} \varrho), \\
& =\text { 0, i.e., } \mathscr{R} \varrho=\varrho .
\end{aligned}
$$

$$
d_{b}\left(\mathscr{T} v_{2 n+1}, \mathcal{S} \varrho\right) \leq \alpha \Lambda_{1}\left(v_{2 n+1}, \varrho\right)+L \min \left\{d_{b}\left(\mathscr{R} v_{2 n+1}, \mathscr{T} v_{2 n+1}\right), d_{b}(\mathscr{R} \varrho, \mathcal{S} \varrho), d_{b}\left(\mathscr{R} v_{2 n+1}, \mathcal{S} \varrho\right), d_{b}\left(\mathscr{R} \varrho, \mathscr{T} v_{2 n+1}\right)\right\}
$$

where

$$
\begin{aligned}
\Lambda_{1}\left(v_{2 n+1}, \varrho\right)= & \max \left\{d_{b}\left(\mathscr{R} v_{2 n+1}, \mathscr{R} \varrho\right), d_{b}\left(\mathscr{R} v_{2 n+1}, \mathscr{T} v_{2 n+1}\right), \frac{d_{b}(\mathscr{R} \varrho, \mathcal{S} \varrho)+d_{b}\left(\mathscr{R} v_{2 n+1}, \mathscr{T} v_{2 n+1}\right)}{2},\right. \\
& \left.\frac{d_{b}\left(\mathscr{T} v_{2 n+1}, \mathscr{R} \varrho\right)+d_{b}\left(\mathscr{R} v_{2 n+1}, \mathcal{S} \varrho\right)}{2 b}\right\} .
\end{aligned}
$$

Letting $n \longrightarrow \infty$ in (62) and using (55) and (56), we get

$$
\begin{aligned}
d_{b}(\varrho, \mathcal{S} \varrho) & \leq \alpha \max \left\{d_{b}(\varrho, \mathcal{S} \varrho), \frac{d_{b}(\mathscr{R} \varrho, \mathcal{S} \varrho)}{2}, \frac{d_{b}(\varrho, \mathscr{R} \varrho)+d_{b}(\varrho, \mathcal{S} \varrho)}{2 b}\right\}, \\
& =\alpha \max \left\{\frac{d_{b}(\varrho, \mathcal{S} \varrho)}{2}, \frac{d_{b}(\varrho, \mathcal{S} \varrho)}{2 b}\right\}, \\
& =\frac{\alpha}{2} d_{b}(\varrho, \mathcal{S} \varrho) \Longrightarrow d_{b}(\varrho, \mathcal{S} \varrho), \\
& =0 \text {, i.e., } \mathcal{S} \varrho=\varrho .
\end{aligned}
$$

Again, $v_{2 n} \prec \delta v_{2 n}$ and $\delta v_{2 n} \longrightarrow \varrho$ as $n \longrightarrow \infty$, so by the assumption we have $v_{2 n} \prec \varrho$ and hence using (39) gives

$$
d_{b}\left(\mathcal{S} v_{2 n}, \mathscr{T} \varrho\right) \leq \alpha \Upsilon_{1}\left(v_{2 n}, \varrho\right)+L \min \left\{d_{b}\left(\mathscr{R} v_{2 n}, \mathcal{S} v_{2 n}\right), d_{b}(\mathscr{R} \varrho, \mathscr{T} \varrho), d_{b}\left(\mathscr{R} v_{2 n}, \mathscr{T} \varrho\right), d_{b}\left(\mathscr{R} \varrho, \mathcal{S} v_{2 n}\right)\right\}
$$


where

$$
Y_{1}\left(v_{2 n}, \varrho\right)=\max \left\{d_{b}\left(\mathscr{R} v_{2 n}, \mathscr{R} \varrho\right), d_{b}\left(\mathscr{R} v_{2 n}, \mathcal{S} v_{2 n}\right), \frac{d_{b}(\mathscr{R} \varrho, \mathscr{T} \varrho)+d_{b}\left(\mathscr{R} v_{2 n}, \mathcal{S} v_{2 n}\right)}{2}, \frac{d_{b}\left(\mathcal{S} v_{2 n}, \mathscr{R} \varrho\right)+d_{b}\left(\mathscr{R} v_{2 n}, \mathscr{T} \varrho\right)}{2 b}\right\}
$$

Letting $n \longrightarrow \infty$ in (65) and using (55) and (56), we have

$$
\begin{aligned}
& d_{b}(\varrho, \mathscr{T} \varrho) \leq \alpha \max \left\{d_{b}(\varrho, \mathscr{R} \varrho), \frac{d_{b}(\mathscr{R} \varrho, \mathscr{T} \varrho)}{2}, \frac{d_{b}(\varrho, \mathscr{R} \varrho)+d_{b}(\varrho, \mathscr{T} \varrho)}{2 b}\right\}, \\
& =\alpha \max \left\{\frac{d_{b}(\varrho, \mathscr{T} \varrho)}{2}, \frac{d_{b}(\varrho, \mathscr{T} \varrho)}{2 b}\right\}, \\
& =\frac{\alpha}{2} d_{b}(\varrho, \mathscr{T} \varrho) \Longrightarrow d_{b}(\varrho, \mathscr{T} \varrho) \text {, } \\
& =0 \text {, } \\
& \text { i.e., } \mathscr{T} \varrho=\varrho \text {. }
\end{aligned}
$$

Therefore, $\delta \varrho=\mathscr{T} \varrho=\mathscr{R} \varrho=\varrho$. Hence $\varrho$ is a common fixed point of $\mathscr{R}, \mathcal{S}$, and $\mathscr{T}$. The proof is similar when (b) holds.
Now, suppose that $\operatorname{CFP}(\mathcal{S}, \mathscr{T}, \mathscr{R})$ is totally ordered. We claim that there is a unique $\operatorname{CFP}(\mathcal{S}, \mathscr{T}, \mathscr{R})$. Assume to the contrary that $\varrho \in \operatorname{CFP}(\mathcal{S}, \mathscr{T}, \mathscr{R})$ and $\sigma \in \operatorname{CFP}(\mathcal{S}, \mathscr{T}, \mathscr{R})$ but $\varrho \neq v$. Using (38) with $v=\varrho$ and $\vartheta=\sigma$, we have

$$
\begin{aligned}
d_{b}(\varrho, \sigma) & =d_{b}(\mathscr{T} \varrho, \mathcal{S} \sigma), \\
& \leq \alpha \Lambda_{1}(\varrho, \sigma)+L \min \left\{d_{b}(\mathscr{R} \varrho, \mathscr{T} \varrho), d_{b}(\mathscr{R} v, \mathcal{S} v), d_{b}(\mathscr{R} \varrho, \mathcal{S} \sigma), d_{b}(\mathscr{R} \sigma, \mathscr{T} \varrho)\right\} \Longrightarrow d_{b}(\varrho, v), \\
& \leq \alpha \Lambda_{1}(\varrho, \sigma),
\end{aligned}
$$

where

$$
\begin{aligned}
\Lambda_{1}(\varrho, \sigma) & =\max \left\{d_{b}(\mathscr{R} \varrho, \mathscr{R} \sigma), d_{b}(\mathscr{R} \varrho, \mathscr{T} \varrho), \frac{d_{b}(\mathscr{R} \sigma, \mathcal{S} \sigma)+d_{b}(\mathscr{R} \varrho, \mathscr{T} \varrho)}{2}, \frac{d_{b}(\mathscr{T} \varrho, \mathscr{R} \sigma)+d_{b}(\mathscr{R} \varrho, \delta \sigma)}{2 b}\right\}, \\
& =\max \left\{d_{b}(\varrho, \sigma), \frac{d_{b}(\varrho, \sigma)}{b}\right\}, \\
& =d_{b}(\varrho, \sigma) .
\end{aligned}
$$

Therefore,

$$
d_{b}(\varrho, \sigma) \leq \alpha d_{b}(\varrho, \sigma)<d_{b}(\varrho, \sigma),
$$

Taking $\mathscr{R}=$ the identity mapping on $\Xi$ in Theorem 4 , we have the following consequence.

a contradiction. Hence, $\varrho=\sigma$. The converse is trivial. 
Corollary 2. Let $\left(\Xi, d_{b}, \prec\right)$ be an ordered right-complete quasi-b-metric space with constant $b \geq 1$. Let the pair of mappings $\mathscr{T}, \mathcal{S}: \Xi \longrightarrow \Xi$ satisfy

$$
\begin{aligned}
& d_{b}(\mathscr{T} \nu, \mathcal{S} \vartheta) \leq \alpha \Lambda_{1}^{\prime}(\nu, \vartheta)+L \min \left\{d_{b}(\nu, \mathscr{T} v), d_{b}(y, \mathcal{S} \vartheta), d_{b}(\nu, \mathcal{S} \vartheta), d_{b}(\vartheta, \mathscr{T} \nu)\right\} \\
& d_{b}(\mathcal{S} \nu, \mathscr{T} \vartheta) \leq \alpha Y_{1}^{\prime}(\nu, \vartheta)+L \min \left\{d_{b}(\nu, \mathcal{S} \nu), d_{b}(y, \mathscr{T} \vartheta), d_{b}(\nu, \mathscr{T} \vartheta), d_{b}(y, \mathcal{S} \nu)\right\}
\end{aligned}
$$

for all comparable $\nu, \vartheta \in \Xi, \alpha \in[0,1), L \geq 0$, and

$$
\begin{aligned}
& \Lambda_{1}^{\prime}(\nu, \vartheta)=\max \left\{d_{b}(\nu, \vartheta), d_{b}(\nu, \mathscr{T} \nu), \frac{d_{b}(y, \mathcal{S} \vartheta)+d_{b}(\nu, \mathscr{T} \nu)}{2}, \frac{d_{b}(\mathscr{T} \nu, \vartheta)+d_{b}(\nu, \mathcal{S} \vartheta)}{2 b}\right\}, \\
& Y_{1}^{\prime}(\nu, \vartheta)=\max \left\{d_{b}(\nu, \vartheta), d_{b}(\nu, \mathcal{S} \nu), \frac{d_{b}(y, \mathscr{T} \vartheta)+d_{b}(\nu, \mathcal{S} \nu)}{2}, \frac{d_{b}(\mathcal{S} \nu, \vartheta)+d_{b}(\nu, \mathscr{T} \vartheta)}{2 b}\right\} .
\end{aligned}
$$

Further, assume that

(i) $\mathscr{T}$ and $\mathcal{S}$ are weakly increasing mapping

(ii) $\mathscr{T}$ and $\mathcal{S}$ are dominating maps

Then $\operatorname{CFP}(\mathcal{S}, \mathscr{T}) \neq \varnothing$. In addition, $\operatorname{CFP}(\mathcal{S}, \mathscr{T})$ is totally ordered if and only if $\mathrm{CFP}(\mathcal{S}, \mathscr{T})$ contains exactly one element.

Now, we provide the following example to authenticate Theorem 4.

Example 2. We consider $\Xi=\{0\} \cup \mathbb{N}$ and define $d_{b}: \Xi \times \Xi \longrightarrow \mathbb{R}$ by

$$
d_{b}(\nu, \vartheta)= \begin{cases}0, & \text { if } \nu=\vartheta \\ \frac{2}{v+\vartheta}, & \text { if any one of } \nu, \vartheta \text { is } 0 \text { and the other is not } 0 \\ \left|\frac{1}{v}-\frac{2}{\vartheta}\right|, & \text { if } \nu \neq \vartheta \text { and } \nu, \vartheta \neq 0 .\end{cases}
$$

Also, we define a relation $\prec$ on $\Xi$ by $\nu \prec \vartheta$ if and only if $\nu \leq \vartheta$. Then, we can easily check that $\left(\Xi, d_{b}, \prec\right)$ is an ordered right-complete quasi- $b$-metric space with constant $b=2$. Next, we define three mappings $\mathscr{T}, \mathcal{S}, \mathscr{R}: \Xi \longrightarrow \Xi$ by

$$
\begin{aligned}
& \mathscr{T} v= \begin{cases}0, & \text { if } v=0 ; \\
20(v+2), & \text { if } v \neq 0,\end{cases} \\
& \mathcal{S} v= \begin{cases}0, & \text { if } v=0 ; \\
40(v+3), & \text { if } v \neq 0,\end{cases} \\
& \mathscr{R} v= \begin{cases}0, & \text { if } v=0 ; \\
v+1, & \text { if } v \neq 0 .\end{cases}
\end{aligned}
$$

Then, clearly $\mathscr{T} \Xi \subseteq \mathscr{R} \Xi$ and $\mathcal{S} \Xi \subseteq \mathscr{R} \Xi$. We choose $\alpha=(19 / 20)$. Now we assume that $\nu, \vartheta \in \Xi$ are arbitrary such that $\mathscr{R} \nu$ and $\mathscr{R} \vartheta$ are comparable. Then the following cases arise:
Case I: let $v, \vartheta \neq 0$ and $\mathscr{R} v \prec \mathscr{R} \vartheta$. First, we assume that $\nu \neq \vartheta$. Then $\quad d_{b}(\mathscr{T} v, \mathcal{S} \vartheta)=(1 / 20)((1 /(\nu+2))-$ $(1 /(\vartheta+3)))$ and $d_{b}(\mathscr{R} v, \mathscr{T} v)=((9 v+19) /(10(\nu+1)$ $(\nu+2)))$. Therefore, we have

$$
d_{b}(\mathscr{T} \nu, \mathcal{S} \vartheta) \leq \alpha d_{b}(\mathscr{R} \nu, \mathscr{T} \nu) \leq \alpha \Lambda_{1}(\nu, \vartheta)
$$

Next, we assume that $v=\vartheta$. Then $d_{b}(\mathscr{T} v, \mathcal{S} \vartheta)=$ $(1 /(20(v+1)(\nu+3)))$ and $d_{b}(\mathscr{R} v, \mathscr{T} v)=((9 v+19) /$ $(10(\nu+1)(\nu+2)))$, and so we have

$$
d_{b}(\mathscr{T} v, \mathcal{S} \vartheta) \leq \alpha d_{b}(\mathscr{R} v, \mathscr{T} v) \leq \alpha \Lambda_{1}(\nu, \vartheta)
$$

In a similar way, we can show that

$$
d_{b}(\mathcal{S} \nu, \mathscr{T} \vartheta) \leq \alpha Y_{1}(\nu, \vartheta)
$$

Case II: let $\nu, \vartheta \neq 0$ and $\mathscr{R} \vartheta \prec \mathscr{R} \nu$. Then, proceeding in the same way as that in Case I, we can show that

$$
\begin{aligned}
& d_{b}(\mathscr{T} \nu, \mathcal{S} \vartheta) \leq \alpha \Lambda_{1}(\nu, \vartheta) \\
& d_{b}(\mathcal{S} \nu, \mathscr{T} \vartheta) \leq \alpha Y_{1}(\nu, \vartheta)
\end{aligned}
$$

Case III: let $v=0$ and $\vartheta \neq 0$. Then $d_{b}(\mathscr{T} v, \mathcal{S} \vartheta)=$ $(1 /(20(\vartheta+3))), \quad d_{b}(\mathcal{S} v, \mathscr{T} \vartheta)=(1 / 10(\vartheta+2)), \quad$ and $d_{b}(\mathscr{R} v, \mathscr{R} \vartheta)=(2 /(\vartheta+1))$. Therefore,

$$
\begin{aligned}
& d_{b}(\mathscr{T} v, \mathcal{S} \vartheta) \leq \alpha d_{b}(\mathscr{R} v, \mathscr{R} \vartheta) \\
& d_{b}(\mathcal{S} v, \mathscr{T} \vartheta) \leq \alpha d_{b}(\mathscr{R} v, \mathscr{R} \vartheta)
\end{aligned}
$$

which implies that

$$
\begin{aligned}
& d_{b}(\mathscr{T} \nu, \mathcal{S} \vartheta) \leq \alpha \Lambda_{1}(\nu, \vartheta) \\
& d_{b}(\mathcal{S} \nu, \mathscr{T} \vartheta) \leq \alpha Y_{1}(\nu, \vartheta)
\end{aligned}
$$


Case IV: let $\vartheta=0$ and $\nu \neq 0$. This case is similar to the previous case and so is omitted.

Case $\mathrm{V}$ : let $v=0=\vartheta$. In this case, it is trivial to check that (38) and (39) hold.

So, combining all the five cases, we see that (38) and (39) hold for all comparable $\mathscr{R} v, \mathscr{R} y \in \Xi$.

Now let $\vartheta \in \mathscr{R}^{-1}(\mathscr{T} \nu)$. Then, we have $\vartheta=20 \nu+39$ if $\nu \neq 0$ and $\vartheta=0$ if $\nu=0$. Therefore, if $\nu \neq 0$, then $\mathscr{T} v=20(\nu+$ 2) and $\mathcal{S} \vartheta=40(20 v+42)$, so $\mathscr{T} v \prec \mathcal{S} \vartheta$. If $v=0$, then $\mathscr{T} v=$ $\mathcal{S} \vartheta=0$ and so $\mathscr{T} v \prec \mathcal{S} \vartheta$. Similarly, for $\vartheta \in \mathscr{R}^{-1}(\mathcal{S} v)$, we can show that $\delta \mathcal{T} \prec \mathscr{T} \vartheta$. Hence $\mathscr{T}$ and $\delta$ are weakly increasing with respect to $\mathscr{R}$. Further, we can easily show that $\mathscr{T}$ and $\mathcal{S}$ are dominating maps; $\mathcal{S}$ and $\mathscr{R}$ are compatible; and $\mathscr{R}$ is continuous. Thus, we can apply Theorem 4 to deduce that $\mathscr{T}, \mathcal{S}$, and $\mathscr{R}$ have a unique common fixed point $\varrho=0$.

\section{Application to Nonlinear Matrix Equations}

In this section, we will apply the common fixed-point results in quasi- $b$-metric spaces of the previous section to obtain variants of the results of Garai and Dey [23] on existence of common solution to systems of NMEs. For other variants on solution to systems of NMEs, one is referred to [24, 25]. For a matrix $\mathscr{A}$, any singular value of $\mathscr{A}$ will be denoted by $s(\mathscr{A})$, and the sum of these values, that is, the trace norm of $\mathscr{A}$, will be denoted by $s^{+}(\mathscr{A})=\|\mathscr{A}\|$. We will use the standard partial order on $H(n)$ given by $\mathscr{A} \geqslant \mathscr{B}$ if and only if $\mathscr{A}-\mathscr{B}$ is a positive semidefinite matrix. We define a function $d_{b}: H(n) \times H(n) \longrightarrow \mathbb{R}$ by

$$
d_{b}(x, y)=\left\{\begin{array}{l}
0, \quad \text { if } \mathscr{X}=\mathscr{Y} ; \\
\left\|\mathscr{X}-\frac{1}{2} \mathcal{Y}\right\|^{2}, \quad \text { if } \mathscr{X} \neq \mathscr{Y} .
\end{array}\right.
$$

Then $\left(H(n), d_{b}, \prec\right)$ is an ordered right-complete quasi$b$-metric space with $b=2$.
Utilizing this quasi- $b$-metric space, we now prove the following theorem regarding the solution (s) of a pair of nonlinear matrix equations.

Theorem 5. Consider the system

$$
\begin{aligned}
& \mathscr{X}=\mathscr{B}_{1}+\sum_{i=1}^{k} \mathscr{A}_{i}^{*} f(\mathscr{X}) \mathscr{A}_{i}, \\
& \mathscr{X}=\mathscr{B}_{2}+\sum_{i=1}^{k} \mathscr{A}_{i}^{*} g(\mathscr{X}) \mathscr{A}_{i},
\end{aligned}
$$

where $\mathscr{B}_{1}, \mathscr{B}_{2} \in P(n), \mathscr{A}_{i} \in M(n), i=1, \ldots, k$, and the operators $f, g: H(n) \longrightarrow H(n)$ are continuous in the trace norm. Let, for some $M, N_{1} \in \mathbb{R}$, and, for any $\mathscr{X} \in P(n)$ with $\|\mathscr{X}\| \leq M, \quad s(f(\mathscr{X})), s(g(\mathscr{X})) \leq N_{1}$ hold for all singular values of $f(\mathscr{X})$ and $g(\mathscr{X})$, respectively. Assume the following:

(1) $\left\|\mathscr{B}_{1}\right\|,\left\|\mathscr{B}_{2}\right\| \leq M-N N_{1} n$, where $\sum_{i=1}^{k}\left\|\mathscr{A}_{i}^{*}\right\|\left\|\mathscr{A}_{i}\right\|=N$

(2) For any $\mathscr{K} \in H(n)$ with $\|\mathscr{K}\| \leq M, \sum_{i=1}^{k} \mathscr{A}_{i}^{*} f(\mathscr{K})$ $\mathscr{A}_{\dot{k}} \geqslant O(O$ stands for null matrix of order $n)$ or $\sum_{i=1}^{k} \mathscr{A}_{i}^{*} g(\mathscr{K}) \mathscr{A}_{i} \geqslant O$ holds

(3) For any $\mathscr{K} \in H(n)$ with $\|\mathscr{K}\| \leq M, \mathscr{K} \prec \mathscr{B}_{1}+\sum_{i=1}^{k}$ $\mathscr{A}_{i}^{*} f(\mathscr{K}) \mathscr{A}_{i}$ and $\mathscr{K} \prec \mathscr{B}_{2}+\sum_{i=1}^{k} \mathscr{A}_{i}^{*} g(\mathscr{K}) \mathscr{A}_{i}$ hold

(4) For any $\mathscr{K} \in H(n)$ with $\|\mathscr{K}\| \leq M$, if $\mathscr{K} \prec \mathscr{B}_{1}+$ $\sum_{i=1}^{k} \mathscr{A}_{i}^{*} f(\mathscr{K}) \mathscr{A}_{i}$ then

$\mathscr{B}_{1}+\sum_{i=1}^{k} \mathscr{A}_{i}^{*} f(\mathscr{K}) \mathscr{A}_{i} \prec \mathscr{B}_{2}+\sum_{i=1}^{k} \mathscr{A}_{i}^{*} g\left(\mathscr{B}_{1}+\right.$

$\left.\sum_{i=1}^{k} \mathscr{A}_{i}^{*} f(\mathscr{K}) \mathscr{A}_{i}\right) \mathscr{A}_{i} \prec \mathscr{B}_{1}+\sum_{i=1}^{k} \mathscr{A}_{i}^{*} f\left(\mathscr{B}_{2}\right.$

$+\sum_{i=1}^{k} \mathscr{A}_{i}^{*} g\left(\mathscr{B}_{1}+\sum_{i=1}^{k} \mathscr{A}_{i}^{*} f(\mathscr{K}) \mathscr{A}_{i}\right) \mathscr{A}_{i}$ hold

(5) There exists $L \geq 0$ such that, for any $s\left(\mathscr{B}_{1}-\mathscr{B}_{2}\right)$,

(a) $s\left(\mathscr{B}_{1}-(1 / 2) \mathscr{B}_{2}\right)+N s(f(\mathscr{X})-(1 / 2) g(\mathscr{Y})) \leq$ $(2 / 3 n)\left[\mathscr{R}_{11}(\mathscr{X}, \mathscr{Y})+L \mathscr{R}_{12}(\mathscr{X}, \mathscr{Y})\right]^{1 / 2}$

holds for all $s(f(\mathscr{X})-(1 / 2) g(\mathscr{Y}))$ and $\mathscr{X}, \mathscr{Y} \in H(n)$ with $\|\mathscr{X}\|,\|\mathscr{Y}\| \leq M, \quad \mathscr{X} \prec \mathscr{Y}$ or $\mathscr{Y} \prec \mathscr{X}$, where

$$
\begin{aligned}
& \mathscr{R}_{11}(\mathscr{X}, \mathscr{Y})=\max \left\{\begin{array}{c}
\left|s^{+}\left(\mathscr{X}-\frac{1}{2} \mathscr{Y}\right)\right|^{2},\left|s^{+}\left(\mathscr{X}-\frac{1}{2} \sum_{i=1}^{k} \mathscr{A}_{i}^{*} f(\mathscr{X}) \mathscr{A}_{i}-\frac{\mathscr{B}_{2}}{2}\right)\right|^{2}, \\
\frac{1}{2}\left[\left|s^{+}\left(\mathscr{Y}-\frac{1}{2} \sum_{i=1}^{k} \mathscr{A}_{i}^{*} g(\mathscr{Y}) \mathscr{A}_{i}-\frac{1}{2} \mathscr{B}_{2}\right)\right|^{2}+\left|s^{+}\left(\mathscr{X}-\frac{1}{2} \sum_{i=1}^{k} \mathscr{A}_{i}^{*} f(\mathscr{X}) \mathscr{A}_{i}-\frac{1}{2} \mathscr{B}_{1}\right)\right|^{2}\right], \\
\frac{1}{4}\left[\left|s^{+}\left(\sum_{i=1}^{k} \mathscr{A}_{i}^{*} f(\mathscr{X}) \mathscr{A}_{i}+\mathscr{B}_{1}-\frac{1}{2} \mathscr{Y}\right)\right|^{2}+\left|s^{+}\left(\mathscr{X}-\frac{1}{2} \sum_{i=1}^{k} \mathscr{A}_{i}^{*} g(\mathscr{Y}) \mathscr{A}_{i}-\frac{1}{2} \mathscr{B}_{2}\right)\right|^{2}\right]
\end{array}\right. \\
& \mathscr{R}_{12}(\mathscr{X}, \mathscr{Y})=\min \left\{\begin{array}{l}
\left|s^{+}\left(\mathscr{X}-\frac{1}{2} \sum_{i=1}^{k} \mathscr{A}_{i}^{*} f(\mathscr{X}) \mathscr{A}_{i}-\frac{1}{2} \mathscr{B}_{1}\right)\right|^{2}\left|s^{+}\left(\mathscr{Y}-\frac{1}{2} \sum_{i=1}^{k} \mathscr{A}_{i}^{*} g(\mathscr{Y}) \mathscr{A}_{i}-\frac{1}{2} \mathscr{B}_{2}\right)\right|^{2}, \\
\left|s^{+}\left(\mathscr{X}-\frac{1}{2} \sum_{i=1}^{k} \mathscr{A}_{i}^{*} g(\mathscr{Y}) \mathscr{A}_{i}-\frac{1}{2} \mathscr{B}_{2}\right)\right|^{2}\left|s^{+}\left(\mathscr{Y}-\frac{1}{2} \sum_{i=1}^{k} \mathscr{A}_{i}^{*} f(\mathscr{X}) \mathscr{A}_{i}-\frac{1}{2} \mathscr{B}_{1}\right)\right|^{2},
\end{array}\right\}
\end{aligned}
$$


(b) $s\left(\mathscr{B}_{1}-(1 / 2) \mathscr{B}_{2}\right)+N s(g(\mathscr{X})-(1 / 2) f(\mathscr{Y})) \leq$ $(2 / 3 n)\left[\mathscr{R}_{21}(\mathscr{X}, \mathscr{Y})+L \mathscr{R}_{22}(\mathscr{X}, \mathscr{Y})\right]^{1 / 2}$ holds for all $s(g(\mathscr{X})-(1 / 2) f(\mathscr{Y}))$ and $\mathscr{X}, \mathscr{Y} \in H(n)$ with $\|\mathscr{X}\|,\|\mathscr{Y}\| \leq M, \mathscr{X} \prec \mathscr{Y}$ or $\mathscr{Y}<\mathscr{X}$, where

$$
\begin{gathered}
\left|s^{+}\left(\mathscr{X}-\frac{1}{2} \mathscr{Y}\right)\right|^{2},\left|s^{+}\left(\mathscr{X}-\frac{1}{2} \sum_{i=1}^{k} \mathscr{A}_{i}^{*} g(\mathscr{X}) \mathscr{A}_{i}-\frac{\mathscr{B}_{2}}{2}\right)\right|^{2} \\
\mathscr{R}_{21}(\mathscr{Y})=\max \left\{\begin{array}{c}
\left|s^{+}\left(\mathscr{Y}-\frac{1}{2} \sum_{i=1}^{k} \mathscr{A}_{i}^{*} f(\mathscr{Y}) \mathscr{A}_{i}-\frac{1}{2} \mathscr{B}_{1}\right)\right|^{2}+\left|s^{+}\left(\mathscr{X}-\frac{1}{2} \sum_{i=1}^{k} \mathscr{A}_{i}^{*} g(\mathscr{X}) \mathscr{A}_{i}-\frac{1}{2} \mathscr{B}_{2}\right)\right|^{2} \\
\frac{1}{4}\left[\left|s^{+}\left(\sum_{i=1}^{k} \mathscr{A}_{i}^{*} g(\mathscr{X}) \mathscr{A}_{i}+\mathscr{B}_{2}-\frac{1}{2} \mathscr{Y}\right)\right|^{+}\left|s^{+}\left(X-\frac{1}{2} \sum_{i=1}^{k} \mathscr{A}_{i}^{*} f(\mathscr{Y}) \mathscr{A}_{i}-\frac{1}{2} \mathscr{B}_{1}\right)\right|^{2}\right]
\end{array}\right. \\
\mathscr{R}_{22}(\mathscr{X}, \mathscr{Y})=\min \left\{\begin{array}{l}
\left|s^{+}\left(\mathscr{X}-\frac{1}{2} \sum_{i=1}^{k} \mathscr{A}_{i}^{*} g(\mathscr{X}) \mathscr{A}_{i}-\frac{1}{2} \mathscr{B}_{2}\right)\right|^{2},\left|s^{+}\left(\mathscr{Y}-\frac{1}{2} \sum_{i=1}^{k} \mathscr{A}_{i}^{*} f(\mathscr{Y}) \mathscr{A}_{i}-\frac{1}{2} \mathscr{B}_{1}\right)\right|^{2}, \mid \\
\left|s^{+}\left(\mathscr{X}-\frac{1}{2} \sum_{i=1}^{k} \mathscr{A}_{i}^{*} f(\mathscr{Y}) \mathscr{A}_{i}-\frac{1}{2} \mathscr{B}_{1}\right)\right|^{2}\left|s^{+}\left(\mathscr{Y}-\frac{1}{2} \sum_{i=1}^{k} \mathscr{A}_{i}^{*} g(\mathscr{X}) \mathscr{A}_{i}-\frac{1}{2} \mathscr{B}_{2}\right)\right|^{2}, \mid
\end{array}\right.
\end{gathered}
$$

Then system (82) has a solution, and if $\widehat{x}$ is a solution of the system, then $\widehat{\mathscr{X}} \in P(n)$ with $\|\widehat{\mathscr{X}}\| \leq M$. Further, the iterative sequence $\left\{\mathscr{X}_{n}\right\}$, where, for $j \geq 0$,

$$
\begin{aligned}
& \mathscr{X}_{2 j+1}=\mathscr{B}_{1}+\sum_{i=1}^{k} \mathscr{A}_{i}^{*} f\left(\mathscr{X}_{2 j}\right) \mathscr{A}_{i}, \\
& \mathscr{X}_{2 j+2}=\mathscr{B}_{2}+\sum_{i=1}^{k} \mathscr{A}_{i}^{*} g\left(\mathscr{X}_{2 j+1}\right) \mathscr{A}_{i},
\end{aligned}
$$

and $\mathscr{X}_{0}$ is an arbitrary element of $H(n)$ satisfying $\left\|\mathscr{X}_{0}\right\| \leq M$, converges to a unique solution of the system, if $X_{j} \prec X_{j+1}$ or $x_{j+1} \prec X_{j}$.

Proof. Let us consider the set $\Xi=\{\mathscr{X} \in H(n):\|\mathscr{X}\| \leq M\}$. Then, $\Xi$ is right-complete with respect to the metric $d_{b}$. For any $\mathscr{X} \in \Xi$, we have

$$
\begin{aligned}
\left\|\mathscr{B}_{1}+\sum_{i=1}^{k} \mathscr{A}_{i}^{*} f(\mathscr{X}) \mathscr{A}_{i}\right\| & \leq\left\|\mathscr{B}_{1}\right\|+\left\|\sum_{i=1}^{k} \mathscr{A}_{i}^{*} f(\mathscr{X}) \mathscr{A}_{i}\right\|, \\
& \leq\left\|\mathscr{B}_{1}\right\|+\sum_{i=1}^{k}\left\|\mathscr{A}_{i}^{*}\right\|\left\|\mathscr{A}_{i}\right\|\|f(\mathscr{X})\|, \\
& =\left\|\mathscr{B}_{1}\right\|+N\|f(\mathscr{X})\| .
\end{aligned}
$$

Since $\|\mathscr{X}\| \leq M$, we have $s(f(\mathscr{X})) \leq N_{1}$ for all singular values $s(f(\mathscr{X}))$ of $f(\mathscr{X})$ so, by summing the $n$ singular values of $f(\mathscr{X})$, we get $\|f(\mathscr{X})\| \leq n N_{1}$. Using this in (86), we get

$$
\left\|\mathscr{B}_{1}+\sum_{i=1}^{k} \mathscr{A}_{i}^{*} f(\mathscr{X}) \mathscr{A}_{i}\right\| \leq\left\|\mathscr{B}_{1}\right\|+n \mathrm{NN}_{1} \leq M .
$$

Similarly, for any $\mathscr{X} \in \Xi$, we can show that

$$
\left\|\mathscr{B}_{2}+\sum_{i=1}^{k} \mathscr{A}_{i}^{*} g(\mathscr{X}) \mathscr{A}_{i}\right\| \leq\left\|\mathscr{B}_{2}\right\|+n \mathrm{NN}_{1} \leq M .
$$

Therefore, the mappings $\mathscr{T}, \mathcal{S}$ defined on $\Xi$ by

$$
\begin{aligned}
\mathscr{T}(\mathscr{X}) & =\mathscr{B}_{1}+\sum_{i=1}^{k} \mathscr{A}_{i}^{*} f(\mathscr{X}) \mathscr{A}_{i}, \\
\mathcal{S}(\mathscr{X}) & =\mathscr{B}_{2}+\sum_{i=1}^{k} \mathscr{A}_{i}^{*} g(X) \mathscr{A}_{i} .
\end{aligned}
$$

for $\mathscr{X} \in \Xi$ are self-maps on $\Xi$. Next, for any $\mathscr{X} \in \Xi$, using assumption (3), we have $\mathscr{X}<\mathscr{T}(\mathscr{X})$ and $\mathscr{X}<\mathcal{S}(\mathscr{X})$. So the mappings $\mathscr{T}, \mathcal{S}$ are dominating mappings. Again, for $\mathscr{X} \in \Xi$, we have $\mathscr{T}(\mathscr{X}) \in \Xi$ and $\mathcal{S}(\mathscr{X}) \in \Xi$. So, for any $\mathscr{X} \in \Xi$, using assumption (4), we have $\mathcal{S}(\mathscr{X}) \prec \mathscr{T} \mathcal{S}(\mathscr{X})$ and $\mathscr{T}(\mathscr{X}) \prec \mathcal{S} \mathscr{T}(\mathscr{X})$. Therefore, $\mathscr{T}, \mathcal{S}$ are weakly increasing mappings. We choose $\alpha=(17 / 18)$. Now let $\mathscr{X}, \mathcal{Y} \in \Xi$ be arbitrary such that either $\mathscr{X} \prec \mathcal{Y}$ or $\mathcal{Y} \prec \mathscr{X}$. Then, we have 


$$
\begin{aligned}
\left\|\mathscr{T}(\mathscr{X})-\frac{1}{2} \mathcal{S}(\mathscr{Y})\right\| & =\left\|\mathscr{B}_{1}+\sum_{i=1}^{k} \mathscr{A}_{i}^{*} f(\mathscr{X}) \mathscr{A}_{i}-\frac{1}{2} \mathscr{B}_{2}-\frac{1}{2} \sum_{i=1}^{k} \mathscr{A}_{i}^{*} g(\mathscr{Y}) \mathscr{A}_{i}\right\|, \\
& \leq\left\|\mathscr{B}_{1}-\frac{\mathscr{B}_{2}}{2}\right\|+\sum_{i=1}^{k}\left\|\mathscr{A}_{i}^{*}\left(f(\mathscr{X})-\frac{1}{2} g(\mathscr{Y})\right) \mathscr{A}_{i}\right\|, \\
& \leq\left\|\mathscr{B}_{1}-\frac{1}{2} \mathscr{B}_{2}\right\|+\sum_{i=1}^{k}\left\|\mathscr{A}_{i}^{*}\right\|\left\|\mathscr{A}_{i}\right\|\left\|f(\mathscr{X})-\frac{1}{2} g(\mathscr{Y})\right\|, \\
& =\left\|\mathscr{B}_{1}-\frac{1}{2} \mathscr{B}_{2}\right\|+N\left\|f(\mathscr{X})-\frac{1}{2} g(\mathscr{Y})\right\| .
\end{aligned}
$$

If $\mathscr{R}_{11}(\mathscr{X}, \mathscr{Y})=\left|s^{+}(\mathscr{X}-(1 / 2) \mathscr{Y})\right|^{2}$, then we have

$$
\begin{aligned}
s\left(\mathscr{B}_{1}-\frac{1}{2} \mathscr{B}_{2}\right)+\mathrm{Ns}\left(f(\mathscr{X})-\frac{1}{2} g(\mathscr{Y})\right) & \leq \frac{2}{3 n}\left[\left|s^{+}\left(\mathscr{X}-\frac{1}{2} \mathscr{Y}\right)\right|^{2}+L \mathscr{R}_{12}(\mathscr{X}, \mathscr{Y})\right]^{1 / 2}, \\
& \Longrightarrow\left\|\mathscr{B}_{1}-\frac{1}{2} \mathscr{B}_{2}\right\|+N\left\|f(\mathscr{X})-\frac{1}{2} g(\mathscr{Y})\right\| \leq \frac{2}{3}\left[\left\|\mathscr{X}-\frac{1}{2} \mathscr{Y}\right\|^{2}+L \mathscr{R}_{12}(\mathscr{X}, \mathscr{Y})\right]^{1 / 2}, \\
& \Longrightarrow\left\|\mathscr{T}(\mathscr{X})-\frac{1}{2} \mathcal{S}(\mathscr{Y})\right\| \leq \frac{2}{3}\left[\left\|\mathscr{X}-\frac{1}{2} \mathscr{Y}\right\|^{2}+L \mathscr{R}_{12}(\mathscr{X}, \mathscr{Y})\right]^{1 / 2}, \\
& \Longrightarrow\left\|\mathscr{T}(\mathscr{X})-\frac{1}{2} \mathcal{S}(\mathscr{Y})\right\|^{2} \leq \frac{4}{9}\left[\left\|\mathscr{X}-\frac{1}{2} \mathscr{Y}\right\|^{2}+L \mathscr{R}_{12}(\mathscr{X}, \mathscr{Y})\right]
\end{aligned}
$$

If $\quad \mathscr{R}_{11}(\mathscr{X}, \mathscr{Y})=\mid s^{+}\left(\mathscr{X}-(1 / 2) \sum_{i=1}^{k} \mathscr{A}_{i}^{*} f(\mathscr{X}) \mathscr{A}_{i}-\right.$ $\left.(1 / 2) \mathscr{B}_{2}\right)\left.\right|^{2}$, then we can similarly show that

Again if

$$
\left\|\mathscr{T}(\mathscr{X})-\frac{1}{2} \mathcal{S}(\mathscr{Y})\right\|^{2} \leq \frac{4}{9}\left[\left\|\mathscr{X}-\frac{1}{2} \mathscr{T}(\mathscr{X})\right\|^{2}+L \mathscr{R}_{12}(\mathscr{X}, \mathscr{Y})\right] .
$$

$$
\mathscr{R}_{11}(\mathscr{X}, \mathscr{Y})=\frac{1}{2}\left[\left|s^{+}\left(\mathscr{Y}-\frac{1}{2} \sum_{i=1}^{k} \mathscr{A}_{i}^{*} g(\mathscr{Y}) \mathscr{A}_{i}-\frac{1}{2} \mathscr{B}_{2}\right)\right|^{2}+\left|s^{+}\left(\mathscr{X}-\frac{1}{2} \sum_{i=1}^{k} \mathscr{A}_{i}^{*} f(\mathscr{X}) \mathscr{A}_{i}-\frac{1}{2} \mathscr{B}_{1}\right)\right|^{2}\right],
$$

or

$$
\mathscr{R}_{11}(\mathscr{X}, \mathscr{Y})=\frac{1}{4}\left[\left|s^{+}\left(\sum_{i=1}^{k} \mathscr{A}_{i}^{*} f(\mathscr{X}) \mathscr{A}_{i}+\mathscr{B}_{1}-\frac{1}{2} \mathscr{Y}\right)\right|^{2}+\left|s^{+}\left(\mathscr{X}-\frac{1}{2} \sum_{i=1}^{k} \mathscr{A}_{i}^{*} g(\mathscr{Y}) \mathscr{A}_{i}-\frac{1}{2} \mathscr{B}_{2}\right)\right|^{2}\right],
$$

then we can show that

$$
\left\|\mathscr{T}(\mathscr{X})-\frac{1}{2} \mathcal{S}(\mathscr{Y})\right\|^{2} \leq \frac{4}{9}\left[\frac{1}{2}\left(\left\|\mathscr{Y}-\frac{1}{2} \mathcal{S}(\mathscr{Y})\right\|^{2}+\left\|\mathscr{X}-\frac{1}{2} \mathscr{T}(\mathscr{X})\right\|^{2}\right)+L \mathscr{R}_{12}(\mathscr{X}, \mathscr{Y})\right],
$$


or

$$
\left\|\mathscr{T}(\mathscr{X})-\frac{1}{2} \mathcal{S}(\mathscr{Y})\right\|^{2} \leq \frac{4}{9}\left[\frac{1}{4}\left(\left\|\mathscr{T}(\mathscr{X})-\frac{1}{2} \mathscr{Y}\right\|^{2}+\left\|\mathscr{X}-\frac{1}{2} \mathcal{S}(\mathscr{Y})\right\|^{2}\right)+L \mathscr{R}_{12}(\mathscr{X}, \mathscr{Y})\right] .
$$

Thus, combining equations (91)-(96) and using the definition of the quasi-b-metric $d_{b}$, we get

$$
\begin{aligned}
& \left\|\mathscr{T}(\mathscr{X})-\frac{1}{2} \mathcal{S}(\mathscr{Y})\right\|^{2} \leq \frac{4}{9} \max \left\{\left\|\mathscr{X}-\frac{1}{2} \mathcal{Y}\right\|^{2},\left\|\mathscr{X}-\frac{1}{2} \mathscr{T}(\mathscr{X})\right\|^{2}, \frac{1}{2}\left(\left\|\mathscr{Y}-\frac{1}{2} \mathcal{S}(\mathscr{Y})\right\|^{2}+\left\|\mathscr{X}-\frac{1}{2} \mathscr{T}(\mathscr{X})\right\|^{2}\right),\right. \\
& \left.\cdot \frac{1}{4}\left(\left\|\mathscr{T}(\mathscr{X})-\frac{1}{2} \mathscr{Y}\right\|^{2}+\left\|\mathscr{X}-\frac{1}{2} \mathcal{S}(\mathscr{Y})\right\|^{2}\right)\right\} \\
& +\frac{4}{9} L \mathscr{R}_{12}(\mathscr{X}, \mathscr{Y}) \Longrightarrow d_{b}(\mathscr{T}(\mathscr{X}), \mathcal{S}(\mathscr{Y})) \\
& \leq \frac{4}{9} \max \left\{d_{b}(\mathscr{X}, \mathscr{Y}), d_{b}(\mathscr{X}, \mathscr{T}(\mathscr{X})), \frac{1}{2}\left(d_{b}(\mathscr{Y}, \mathcal{S}(\mathscr{Y}))+d_{b}(\mathscr{X}, \mathscr{T}(\mathscr{X}))\right), \frac{1}{2 b}\left(d_{b}(\mathscr{T}(\mathscr{X}), \mathscr{Y})+d_{b}(\mathscr{X}, \mathcal{S}(\mathscr{Y}))\right)\right\}, \\
& +\frac{4}{9} L \mathscr{R}_{12}(\mathscr{X}, \mathscr{Y}) \Longrightarrow d_{b}(\mathscr{T}(\mathscr{X}), \mathcal{S}(\mathscr{Y})) \\
& \leq \alpha \max \left\{d_{b}(\mathscr{X}, \mathscr{Y}), d_{b}(\mathscr{X}, \mathscr{T}(\mathscr{X})), \frac{d_{b}(\mathscr{Y}, \mathcal{S}(\mathscr{Y}))+d_{b}(\mathscr{X}, \mathscr{T}(\mathscr{X}))}{2}, \frac{d_{b}(\mathscr{T}(\mathscr{X}), \mathscr{Y})+d_{b}(\mathscr{X}, \mathcal{S}(\mathscr{Y}))}{2 b}\right\} \\
& +\frac{4}{9} L \mathscr{R}_{12}(\mathscr{X}, \mathscr{Y}) \Longrightarrow d_{b}(\mathscr{T}(\mathscr{X}), \mathcal{S}(\mathscr{Y})) \\
& \leq \alpha \Lambda_{1}^{\prime}(\mathscr{X}, \mathscr{Y})+L \min \left\{d_{b}(\mathscr{X}, \mathscr{T}(\mathscr{X})), d_{b}(\mathscr{Y}, \mathcal{S}(\mathscr{Y})), d_{b}(\mathscr{X}, \mathcal{S}(\mathscr{Y})), d_{b}(\mathscr{Y}, \mathscr{T}(\mathscr{X}))\right\} .
\end{aligned}
$$

In a similar way, we can show that

$$
d_{b}(\mathcal{S}(\mathscr{X}), \mathscr{T}(\mathscr{Y})) \leq \alpha Y_{1}^{\prime}(\mathscr{X}, \mathscr{Y})+L \min \left\{d_{b}(\mathscr{X}, \mathcal{S}(\mathscr{X})), d_{b}(\mathscr{Y}, \mathscr{T}(\mathscr{Y})), d_{b}(\mathscr{X}, \mathscr{T}(\mathscr{Y})), d_{b}(\mathscr{Y}, \mathcal{S}(\mathscr{X}))\right\}
$$

Hence, by Corollary 2, it is implied that the pair of mappings $\mathscr{T}, \mathcal{S}$ has a common fixed point in $\Xi$, say $\widehat{\mathscr{X}}$. So $\widehat{\mathscr{X}}$ is a solution to system (82). By assumption (2), we see that any solution of system (82) must be positive definite, and since $\widehat{\mathscr{X}} \in \Xi$, we have $\|\widehat{\mathscr{X}}\| \leq M$. Again, by the order preserving (or order reversing) of the successive elements of the sequence $\left\{\mathscr{X}_{n}\right\}$ converging to $\widehat{\mathscr{X}}$, we see that system (82) cannot have more than one solution. Thus, system (82) has a unique solution $\widehat{\mathscr{X}} \in \Xi$ with $\widehat{\mathscr{X}} \in P(n)$ and $\|\widehat{\mathscr{X}}\| \leq M$.
Now we present an example in order to validate the above theorem.

Example 3. Consider system (82) for $k=2$ and $n=4$, with $f(\mathscr{X})=\mathscr{X}^{0.2}$ and $g(\mathscr{X})=\mathscr{X}^{0.3}$; that is,

$$
\begin{aligned}
& \mathscr{X}=\mathscr{B}_{1}+\mathscr{A}_{1}^{*} \mathscr{X}^{0.2} \mathscr{A}_{1}+\mathscr{A}_{2}^{*} \mathscr{X}^{0.2} \mathscr{A}_{2}, \\
& \mathscr{X}=\mathscr{B}_{2}+\mathscr{A}_{1}^{*} \mathscr{X}^{0.3} \mathscr{A}_{1}+\mathscr{A}_{2}^{*} \mathscr{X}^{0.3} \mathscr{A}_{2},
\end{aligned}
$$


where

$$
\begin{aligned}
& \mathscr{A}_{1}= {\left[\begin{array}{llll}
0.50241365 & 0.80309106 & 0.74147738 & 1.0008952 \\
0.80309106 & 1.51926454 & 1.55315745 & 1.68489937 \\
0.74147738 & 1.55315745 & 2.24960894 & 2.07448592 \\
1.0008952 & 1.68489937 & 2.07448592 & 2.41733981
\end{array}\right], } \\
& \mathscr{A}_{2}=\left[\begin{array}{llll}
0.57489671 & 1.04590664 & 0.9683583 & 0.96680342 \\
1.04590664 & 2.5508309 & 1.90415829 & 2.05691324 \\
0.9683583 & 1.90415829 & 2.09247523 & 1.61626038 \\
0.96680342 & 2.05691324 & 1.61626038 & 1.94523108
\end{array}\right], \\
& \mathscr{B}_{1}=\left[\begin{array}{llll}
1.76535051 & 1.19912087 & 1.6487913 & 2.01599104 \\
1.19912087 & 2.02881606 & 1.62300354 & 1.7232668 \\
1.6487913 & 1.62300354 & 1.879647 & 2.10149668 \\
2.01599104 & 1.7232668 & 2.10149668 & 2.44522015
\end{array}\right], \\
& \mathscr{B}_{2}=\left[\begin{array}{lllll}
-10.643334057848985 & -23.874210038032118 & -23.420523624735278 & -23.908390438513909 \\
-23.874210038032118 & -48.926958696625590 & -48.676364597578200 & -50.163509103606913 \\
-23.420523624735285 & -48.676364597578200 & -49.153540195555415 & -50.862360333937076 \\
-23.908390438513909 & -50.163509103606913 & -50.862360333937083 & -52.535431964591425
\end{array}\right] .
\end{aligned}
$$

After calculations, we get $\left\|\mathscr{B}_{1}\right\|=8.11903372,\left\|\mathscr{B}_{2}\right\|=$ 163.0367049758027, $\quad N=96.052515868700326, \quad N_{1}=$ 6.183163814689865, and $L=3$.
Let $M=1.038194798043281 \times 10^{3}$. The conditions of Theorem (99) can be checked numerically, taking various special values for matrices involved. For example, they can be tested (and verified to be true) for

$$
\begin{aligned}
\mathscr{X} & =\left[\begin{array}{llll}
2.9958423 & 2.77146661 & 2.54547199 & 2.33145834 \\
2.77146661 & 2.70100098 & 2.20736932 & 2.16196261 \\
2.54547199 & 2.2073693 & 2.40532238 & 2.03543311 \\
2.33145834 & 2.16196261 & 2.03543311 & 1.87555389
\end{array}\right], \\
\mathscr{Y} & =\left[\begin{array}{lllll}
7.91454201 & 7.07030238 & 7.30633152 & 6.80248367 \\
7.07030238 & 7.64595944 & 6.49795464 & 6.58540998 \\
7.30633152 & 6.49795464 & 7.50461026 & 6.76707205 \\
6.80248367 & 6.58540998 & 6.76707205 & 6.5164748
\end{array}\right], \\
\mathscr{K} & =\left[\begin{array}{llll}
0.244895463279295 & 0.446163048925549 & 0.536423236239700 & 0.581367922915251 \\
0.446163048925549 & 0.820430394467024 & 0.994442779832152 & 1.065859543793928 \\
0.536423236239700 & 0.994442779832152 & 1.232631898474834 & 1.304057463080299 \\
0.581367922915251 & 1.065859543793928 & 1.304057463080299 & 1.398770067769852
\end{array}\right],
\end{aligned}
$$

where,

$$
\begin{aligned}
\|\mathscr{X}\| & =9.97771955 \\
\|\mathscr{Y}\| & =29.58158651, \\
\|\mathscr{K}\| & =3.696727823991004 .
\end{aligned}
$$

To see the convergence of the sequence $\left\{\mathscr{X}_{n}\right\}$ defined in (85), we start with initial values, 


$$
\mathscr{X}_{0}=\left[\begin{array}{lllll}
2.046172298403625 & 3.404527441644321 & 2.953394842790926 & 2.942886557017088 \\
3.404527441644321 & 6.364569270588873 & 5.449300388429051 & 5.499469136857805 \\
2.953394842790926 & 5.449300388429052 & 4.867308622636568 & 4.789598061118900 \\
2.942886557017088 & 5.499469136857805 & 4.789598061118900 & 4.890358774128345
\end{array}\right],
$$

with $\left\|\mathscr{X}_{0}\right\|=18.168408965757408$,

$$
\mathscr{U}_{0}=\left[\begin{array}{lllll}
10.293364321414856 & 12.838879399150237 & 13.601326678824901 & 13.518191855227126 \\
12.838879399150237 & 17.873328031056058 & 19.385378724242695 & 19.662286105737081 \\
13.601326678824901 & 19.385378724242695 & 22.111211997385734 & 22.244839258390460 \\
13.518191855227126 & 19.662286105737081 & 22.244839258390460 & 22.959343292059014
\end{array}\right],
$$

with $\left\|\mathscr{U}_{0}\right\|=73.237247641915687$,

$$
\mathscr{V}_{0}=\left[\begin{array}{cccc}
8.383022 & 9.0642937 & 9.05273 & 9.8551909 \\
9.0642937 & 9.95876169 & 10.0729775 & 10.8741133 \\
9.05273 & 10.0729775 & 10.424364 & 11.1353359 \\
9.8551909 & 10.8741133 & 11.1353359 & 12.02731569
\end{array}\right] \text {, }
$$

(105) with $\left\|\mathscr{V}_{0}\right\|=40.793463379999992$ and, after 20 iterations, we have the following approximation of the unique positive definite solution of system (99):

$$
\widehat{\mathscr{X}} \approx \mathscr{X}_{20}=\left[\begin{array}{llll}
18.838973862713779 & 35.660700810728500 & 36.046104062493825 & 37.656448431121959 \\
35.660700810728507 & 72.239396779986436 & 70.690005868946088 & 73.105053026182759 \\
36.046104062493811 & 70.690005868946088 & 72.345460354729028 & 74.914965627691828 \\
37.656448431121959 & 73.105053026182759 & 74.914965627691828 & 78.209549859295251
\end{array}\right],
$$

with $\|\widehat{\mathscr{X}}\|=2.416333808567245 \times 10^{2}$.

$$
\widehat{\mathscr{U}} \approx \mathscr{U}_{20}=\left[\begin{array}{lllll}
18.838973862713768 & 35.660700810728478 & 36.046104062493797 & 37.656448431121945 \\
35.660700810728478 & 72.239396779986379 & 70.690005868946031 & 73.105053026182688 \\
36.046104062493797 & 70.690005868946059 & 72.345460354728971 & 74.914965627691771 \\
37.656448431121930 & 73.105053026182702 & 74.914965627691771 & 78.209549859295194
\end{array}\right],
$$

with $\|\widehat{\mathscr{U}}\|=2.416333808567243 \times 10^{2}$.

$$
\hat{\mathscr{V}} \approx \mathscr{V}_{20}=\left[\begin{array}{lllll}
18.838973862713765 & 35.660700810728486 & 36.046104062493811 & 37.656448431121930 \\
35.660700810728471 & 72.239396779986379 & 70.690005868946031 & 73.105053026182702 \\
36.046104062493782 & 70.690005868946031 & 72.345460354728971 & 74.914965627691785 \\
37.656448431121930 & 73.105053026182702 & 74.914965627691743 & 78.209549859295180
\end{array}\right],
$$


with $\|\hat{\mathscr{V}}\|=2.416333808567243 \times 10^{2}$. Also, the elements of each sequence are order preserving. The graphical representation of convergence is shown below.

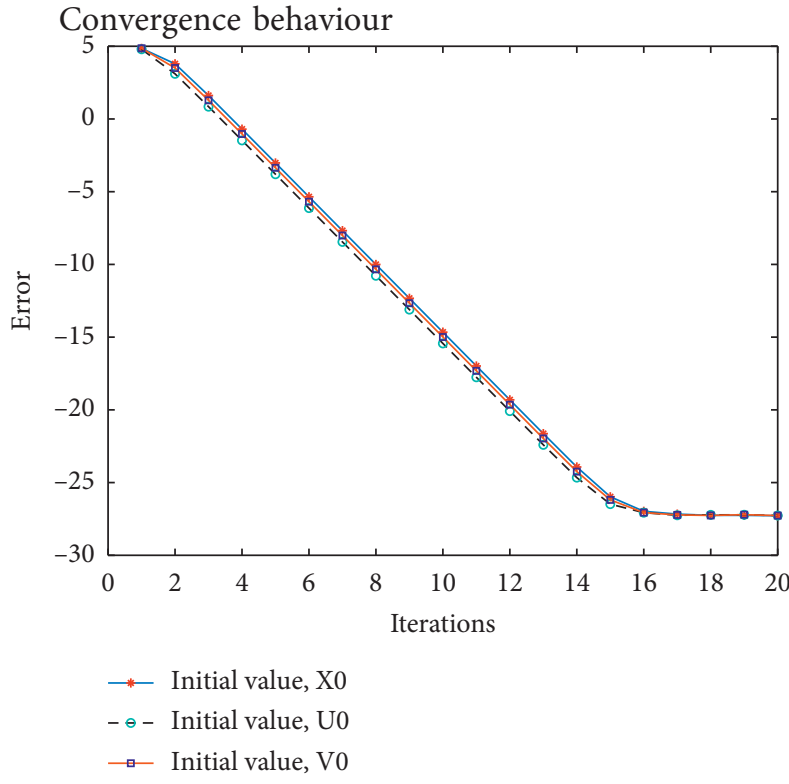

\section{Application to Nonlinear Fractional Differential Equations}

Consider a pair of nonlinear fractional differential equations (FDEs, in short):

$$
\begin{aligned}
& D^{\beta}(\vartheta(t))+\hbar_{1}(t, \vartheta(t))=0, \\
& D^{\beta}(\vartheta(t))+\hbar_{2}(t, \vartheta(t))=0,
\end{aligned}
$$

where $0 \leq t \leq 1,1<\beta$, with the two-point boundary conditions

$$
\begin{aligned}
& \vartheta(0)=0, \\
& \vartheta(1)=0,
\end{aligned}
$$

where $\hbar_{1}, \hbar_{2}: J=[0,1] \times \mathbb{R} \longrightarrow \mathbb{R}$ is a continuous function.

The Caputo derivative of fractional-order $\beta$ is defined as

$$
\begin{array}{r}
{ }^{c} D^{\beta}(\varrho(t))=\frac{1}{\Gamma(n-\beta)} \int_{0}^{t}(t-s)^{n-\beta-1} \varrho^{(n)}(s) \mathrm{d} s, \\
(n-1<\beta<n, n=[\beta]+1),
\end{array}
$$

where $\varrho:[0, \infty) \longrightarrow \mathbb{R}$ is a continuous function, $[\beta]$ denotes the integer part of the positive real number $\beta$, and $\Gamma$ is the gamma function.

The Riemann-Liouville fractional derivative of order $\beta$ for a continuous function $\varrho(t)$ is defined by

$$
\begin{array}{r}
D^{\beta}(\varrho(t))=\frac{1}{\Gamma(n-\beta)}\left(\frac{\mathrm{d}}{\mathrm{d} t}\right)^{n} \int_{0}^{t} \frac{\varrho(s)}{(t-s)^{\beta-n-1}} \mathrm{~d} s, \\
(n-1<\beta<n, n=[\beta]+1),
\end{array}
$$

provided the right-hand side is point-wise defined on $(0, \infty)$

The FDEs (109) are equivalent to the integral equation $\vartheta(t)=\int_{0}^{1} \mathscr{B}(t, \zeta) \hbar_{j}(\zeta, \vartheta(\zeta)) d \zeta, \quad$ for all $t \in J, j=1,2$,

where the Green function is

$$
\mathscr{B}(t, \zeta)= \begin{cases}(t(1-\zeta))^{\beta-1}-(t-\zeta)^{\beta-1}, & 0 \leq \zeta<t \leq 1 \\ \frac{(t(1-\zeta))^{\alpha-1}}{\Gamma(\beta)}, & 0 \leq t \leq \zeta \leq 1 .\end{cases}
$$

Following [4], consider that $\mathscr{X}=C([0,1], \mathbb{R})$ endowed with the metric

$$
d_{b}(\nu, \vartheta)= \begin{cases}\left\|(\nu-\vartheta)^{2}\right\|_{\infty}+\|\nu\|_{\infty}, & \text { for all } \nu, \vartheta \in \mathscr{X} \text { with } \nu \neq \vartheta \\ 0, & \text { otherwise. }\end{cases}
$$

is a right-complete quasi- $b$-metric space with $b=2$, where

$$
\|\vartheta\|_{\infty}=\max _{t \in J}|\vartheta(t)|
$$

is the usual supremum norm. It is noticed that $\left(\mathscr{X}, d_{b}, 2, \prec\right)$ is a right-complete ordered quasi- $b$-metric space, where $\prec$ denotes $v \prec \vartheta$ if $\nu(t) \leq \vartheta(t)$ for all $t \in J$.

Theorem 6. Let $\mathscr{T}, \mathcal{S}: \mathscr{X} \longrightarrow \mathscr{X}$ be two operators defined by

$$
\begin{aligned}
& \mathscr{T} \vartheta(t)=\int_{0}^{1} \mathscr{B}(t, \zeta) \hbar_{1}(\zeta, \vartheta(\zeta)) \mathrm{d} \zeta \\
& \mathcal{S} \vartheta(t)=\int_{0}^{1} \mathscr{B}(t, \zeta) \hbar_{2}(\zeta, \vartheta(\zeta)) \mathrm{d} \zeta
\end{aligned}
$$

where $t \in J$. Assume the following:

(i) $\hbar_{1}, \hbar_{2}: J \times \mathbb{R} \longrightarrow \mathbb{R}$ is a continuous function, which is nondecreasing in the second variable

(ii) For all $t \in J$,

$$
\begin{aligned}
& \hbar_{1}(t, \vartheta(t)) \leq \hbar_{2}\left(t, \int_{0}^{1} \mathscr{B}(t, \tau) \hbar_{1}(\tau, \vartheta(\tau)) \mathrm{d} \tau\right), \\
& \hbar_{2}(t, \vartheta(t)) \leq \hbar_{1}\left(t, \int_{0}^{1} \mathscr{B}(t, \tau) \hbar_{2}(, \vartheta(\tau)) \mathrm{d} \tau\right),
\end{aligned}
$$

(iii) For all $t \in J$,

$$
\vartheta(t) \leq \int_{0}^{1} \mathscr{B}(t, \zeta) \hbar_{j}(\zeta, \vartheta(\zeta)) \mathrm{d} \zeta
$$

(iv) For all $t, s \in J, i \neq j=1,2$, 


$$
\begin{aligned}
0 \leq \hbar_{i}(t, v(s))-\hbar_{j}(t, \vartheta(s)) \leq \frac{1}{b}(\nu(s)-\vartheta(s)) & \\
\nu, \vartheta \in \mathscr{X}, \nu & <\vartheta
\end{aligned}
$$$$
\left|\hbar_{j}(t, v(s))\right| \leq \frac{1}{b} \nu(s) .
$$

Then, the integral equations (113) have a solution $u^{*} \in C(J, \mathbb{R})$.

Proof. Using (II), for all $t \in J$, we have

$$
\begin{aligned}
\mathscr{T} \mathcal{V}(t) & =\int_{0}^{1} \mathscr{B}(t, s) \hbar_{1}(s, \vartheta(s)) \mathrm{d} s, \\
& \leq \int_{0}^{1} \mathscr{B}(t, s) \hbar_{2}\left(s, \int_{0}^{1} \mathscr{B}(s, \tau) \hbar_{1}(\tau, \mathcal{\vartheta}(\tau)) \mathrm{d} \tau\right) \mathrm{d} s, \\
& =\int_{0}^{1} \mathscr{B}(t, s) \hbar_{2}(s, \mathscr{T} \vartheta(s)) \mathrm{d} s, \\
& =\mathcal{S} \mathscr{T} \vartheta(t) .
\end{aligned}
$$

Similarly,

$$
\begin{aligned}
\mathcal{S} \vartheta(t) & =\int_{0}^{1} \mathscr{B}(t, s) \hbar_{2}(s, \vartheta(s)) \mathrm{d} s, \\
& \leq \int_{0}^{1} \mathscr{B}(t, s) \hbar_{1}\left(s, \int_{0}^{1} \mathscr{B}(s, \tau) \hbar_{2}(\tau, \vartheta(\tau)) \mathrm{d} \tau\right) \mathrm{d} s, \\
& =\int_{0}^{1} \mathscr{B}(t, s) \hbar_{1}(s, \mathcal{S} \vartheta(s)) \mathrm{d} s, \\
& =\mathscr{T} \mathcal{S} \vartheta(t) .
\end{aligned}
$$

Then, we have $\mathscr{T} \mathcal{\prec}<\mathscr{S T} \vartheta$ and $\mathcal{S} \vartheta<\mathscr{T} \mathcal{S} \vartheta$ for all $\vartheta \in C(J, \mathbb{R})$. This implies that $\mathscr{T}$ and $\mathcal{S}$ are weakly increasing. Using (III), for all $t \in J, \mathscr{T}$ and $\delta$ are dominating operators.

To check contraction conditions, we start with

$$
\begin{aligned}
\int_{0}^{1} \mathscr{B}(t, \zeta) \mathrm{d} \zeta= & \int_{0}^{c}\left[(t(1-\zeta))^{\beta-1}-(t-\zeta)^{\beta-1}\right] \mathrm{d} \zeta \\
& +\int_{c}^{1} \frac{(t(1-\zeta))^{\alpha-1}}{\Gamma(\beta)} \mathrm{d} \zeta, \\
= & c^{\beta}+\frac{1}{\Gamma(\beta)}[c(1-c)]^{\beta},
\end{aligned}
$$

that is, for $c \in(0,1)$,

$$
\int_{0}^{1} \mathscr{B}(t, \zeta) \mathrm{d} \zeta=c^{\beta}+\frac{1}{\Gamma(\beta)}[c(1-c)]^{\beta}=\lambda, \text { say. }
$$

Now

$$
|\mathscr{T} \vartheta(t)| \leq \int_{0}^{t} \mathscr{B}(t, s)\left|\hbar_{1}(t, \vartheta(s))\right| \mathrm{d} s \leq \int_{0}^{1} \mathscr{B}(t, s) \frac{\lambda}{b}|\vartheta(s)| \mathrm{d} s
$$

$$
=\frac{\lambda}{b}\|\vartheta\|_{\infty}
$$

$|\mathcal{S} \vartheta(t)| \leq \int_{0}^{t} \mathscr{B}(t, s)\left|\hbar_{2}(t, \vartheta(s))\right| \mathrm{d} s \leq \int_{0}^{1} \mathscr{B}(t, s) \frac{\lambda}{b}|\vartheta(s)| \mathrm{d} s$

$$
=\frac{\lambda}{b}\|\vartheta\|_{\infty}
$$

Also

$$
\begin{aligned}
|\mathscr{T} v(t)-\mathcal{S} \vartheta(t)| & \leq \int_{0}^{1}\left|\hbar_{1}(t, v(s))-\hbar_{2}(t, \vartheta(s))\right| \mathrm{d} s \\
& \leq \int_{0}^{1} \mathscr{B}(t, s) \frac{1}{b}|\nu(s)-\vartheta(s)| \mathrm{d} s=\frac{1}{b} \int_{0}^{1} \mathscr{B}(t, s) \sqrt{(\nu(s)-\vartheta(s))^{2}} \mathrm{~d} s, \\
& \leq \frac{\lambda}{b} \sqrt{\left\|(\nu-\vartheta)^{2}\right\|_{\infty}}, \\
|\mathcal{S} \nu(t)-\mathscr{T} \vartheta(t)| & \leq \int_{0}^{1}\left|\hbar_{2}(t, \nu(s))-\hbar_{1}(t, \vartheta(s))\right| \mathrm{d} s \leq \int_{0}^{1} \mathscr{B}(t, s) \frac{1}{b}|\nu(s)-\vartheta(s)| \mathrm{d} s, \\
& =\frac{1}{b} \int_{0}^{1} \mathscr{B}(t, s) \sqrt{(\nu(s)-\vartheta(s))^{2}} \mathrm{~d} s \leq \frac{\lambda}{b} \sqrt{\left\|(\nu-\vartheta)^{2}\right\|_{\infty}} .
\end{aligned}
$$


Therefore, we get

$$
\begin{gathered}
\|\mathscr{T} \vartheta\|_{\infty} \leq \frac{\lambda}{b}\|\vartheta\|_{\infty}, \\
\left\|(\mathscr{T} v-\mathcal{S} \vartheta)^{2}\right\|_{\infty} \leq \frac{\lambda^{2}}{b^{2}}\left\|(\nu-\vartheta)^{2}\right\|_{\infty}, \\
\|\mathcal{S} \vartheta\|_{\infty} \leq \frac{\lambda}{b}\|\vartheta\|_{\infty}, \\
\left\|(\mathcal{S} v-\mathscr{T} \vartheta)^{2}\right\|_{\infty} \leq \frac{\lambda^{2}}{b^{2}}\left\|(\nu-\vartheta)^{2}\right\|_{\infty} .
\end{gathered}
$$

Hence

$$
\begin{aligned}
& d_{b}(\mathscr{T} v, \mathcal{S} \vartheta) \leq \frac{\lambda}{b} d_{b}(\nu, \vartheta), \\
& d_{b}(\mathcal{S} \nu, \mathcal{T} \vartheta) \leq \frac{\lambda}{b} d_{b}(\nu, \vartheta),
\end{aligned}
$$

for all comparable $\nu, \vartheta \in \mathscr{X}$ such that $\mathscr{T} \nu \neq \mathcal{S} \vartheta$. Also, it is an obvious fact that the above inequality holds true if $\mathscr{T} v=\mathcal{S} \vartheta$. Therefore, for $\alpha=(\lambda / b)$ and $L=0$, Corollary 2 implies that there is a unique common fixed point $u^{*} \in \mathscr{X}$ of the operators $(\mathscr{T}, \mathcal{S})$; that is, $u^{*}$ is also a solution to the integral equation (117) and the FDEs (109). To see the uniqueness of solution, let $\omega^{*} \in \mathscr{X}$ be another solution of integral equation (117); then, using condition (II), we have

$$
\begin{aligned}
u^{*}(t)-\omega^{*}(t) & =\left|\int_{0}^{1} \mathscr{B}(t, \zeta) \hbar_{1}\left(\zeta, u^{*}(\zeta)\right) \mathrm{d} \zeta-\int_{0}^{1} \mathscr{B}(t, \zeta) \hbar_{2}\left(\zeta, \omega^{*}(\zeta)\right) \mathrm{d} \zeta\right|, \\
& \leq \int_{0}^{1} \mathscr{B}(t, \zeta)\left|\hbar_{1}\left(\zeta, u^{*}(\zeta)\right)-\hbar_{2}\left(\zeta, \omega^{*}(\zeta)\right)\right| \mathrm{d} \zeta, \\
& \leq \frac{1}{b} \int_{0}^{1} \mathscr{B}(t, \zeta)\left|u^{*}(\zeta)-\omega^{*}(\zeta)\right| \mathrm{d} \zeta, \\
& \leq \frac{\lambda}{b}\left|u^{*}(\zeta)-\omega^{*}(\zeta)\right|,
\end{aligned}
$$

that is, $\left\|u^{*}-\omega^{*}\right\|_{\infty} \leq(\lambda / b)\left\|u^{*}-\omega^{*}\right\|_{\infty}$, a contradiction, as $\lambda \in(0,1), b \geq 1$.

\section{Concluding Remark}

We obtain some common fixed-point results for a pair of mappings and also for a triplet of mappings in quasi$b$-metric spaces using the left-and right-completeness in the absence of commutativity condition. We utilize these results to obtain solutions of a pair of nonlinear matrix equations and a pair of fractional differential equations.

\section{Data Availability}

No data were used to support this study.

\section{Conflicts of Interest}

The authors declare that they have no conflicts of interest.

\section{Acknowledgments}

The first and second authors are thankful to SERB, India, for providing fund under the project CRG/2018/000615. The third author is thankful to CSIR, New Delhi, India, for their financial support under CSIR-SRF fellowship scheme (Award no. 09/973 (0018)/2017-EMR-I).

\section{References}

[1] S. Czerwik, "Contraction mappings in b-metric spaces," Acta Mathematica et Informatica Universitatis Ostraviensis, vol. 1, no. 1, pp. 5-11, 1993.

[2] M. H. Shah and N. Hussain, "Nonlinear contractions in partially ordered quasi $b$-metric spaces," Communications of the Korean Mathematical Society, vol. 27, no. 1, pp. 117-128, 2012.

[3] H. Aydi, M. Jellali, M. Jellali, and E. Karapınar, "On fixed point results for a-implicit contractions in quasi-metric spaces and consequences," Nonlinear Analysis: Modelling and Control, vol. 21, no. 1, pp. 40-56, 2016.

[4] N. Hussain, C. Vetro, and F. Vetro, "Fixed point results for $\alpha$-implicit contractions with application to integral equations," Nonlinear Analysis: Modelling and Control, vol. 21, no. 3, pp. 362-378, 2016.

[5] M. Turinici, "Abstract comparison principles and multivariable gronwall-bellman inequalities," Journal of Mathematical Analysis and Applications, vol. 117, no. 1, pp. 100-127, 1986.

[6] M. Turinici, "Fixed points for monotone iteratively local contractions," Demonstratio Mathematica, vol. 19, no. 1, pp. 171-180, 1986.

[7] J. Matkowski, "Integrable solutions of functional equations," Dissertationes Mathematicae, vol. 127, p. 68, 1975.

[8] J. Matkowski, "Fixed point theorems for mappings with a contractive iterate at a point," Proceedings of the American Mathematical Society, vol. 62, no. 2, p. 344, 1977.

[9] A. C. M. Ran and M. C. B. Reurings, "A fixed point theorem in partially ordered sets and some applications to matrix 
equations," Proceedings of the American Mathematical Society, vol. 132, no. 5, pp. 1435-1443, 2004.

[10] J. J. Nieto and R. Rodríguez-López, "Contractive mapping theorems in partially ordered sets and applications to ordinary differential equations," Order, vol. 22, no. 3, pp. 223-239, 2005.

[11] J. J. Nieto and R. Rodríguez-López, "Existence and uniqueness of fixed point in partially ordered sets and applications to ordinary differential equations," Acta Mathematica Sinica, English Series, vol. 23, no. 12, pp. 2205-2212, 2007.

[12] F. Gu and W. Shatanawi, "Some new results on common coupled fixed points of two hybrid pairs of mappings in partial metric spaces," Journal of Nonlinear Functional Analysis, vol. 2019, p. 13, 2019.

[13] A. Latif, T. Nazir, and M. Abbas, "Stability of fixed points in generalized metric spaces," Journal of Nonlinear and Variational Analysis, vol. 2, no. 3, pp. 287-294, 2018.

[14] S. K. Malhotra, S. Prakash, and S. Shukla, "A generalization of Nadler theorem in cone $b$-metric spaces over Banach algebras," Communications in Optimization Theory, vol. 2019, p. 10, 2019.

[15] H. K. Nashine, B. Samet, and C. Vetro, "Monotone generalized nonlinear contractions and fixed point theorems in ordered metric spaces," Mathematical and Computer Modelling, vol. 54, no. 1-2, pp. 712-720, 2011.

[16] L. Cirić, N. Cakić, M. Rajović, and J. S. Ume, "Monotone generalized nonlinear contractions in partially ordered metric spaces," Fixed Point Theory and Applications, vol. 2008, Article ID 131294, 2008.

[17] H. K. Nashine and I. Altun, "New fixed point results for maps satisfying implicit relations on ordered metric spaces and application," Applied Mathematics and Computation, vol. 240, no. 1, pp. 259-272, 2014.

[18] H. K. Nashine and B. Samet, "Fixed point results for mappings satisfying $(\psi, \varphi)$-weakly contractive condition in partially ordered metric spaces," Nonlinear Analysis, vol. 74, no. 4, pp. 2201-2209, 2011.

[19] M. Abbas, T. Nazir, and S. Radenović, "Common fixed points of four maps in partially ordered metric spaces," Applied Mathematics Letters, vol. 24, no. 9, pp. 1520-1526, 2011.

[20] C. Vetro, "Common fixed points in ordered banach spaces," Le Matematiche, vol. 63, no. 2, pp. 93-100, 2008.

[21] H. K. Nashine, B. Samet, and J. K. Kim, "Fixed point results for contractions involving generalized altering distances in ordered metric spaces," Fixed Point Theory and Applications, vol. 2011, no. 1, pp. 1-16, 2011.

[22] T. Suzuki, "Basic inequality on a $b$-metric space and its applications," Journal of Inequalities and Applications, vol. 2017, p. 256, 2017.

[23] H. Garai and L. K. Dey, "Common solution to a pair of nonlinear matrix equations via fixed point results," Journal of Fixed Point Theory and Applications, vol. 21, no. 2, p. 61, 2019.

[24] A. Bera, L. K. Dey, H. Garai, and S. Raha, "Common fixed points via asymptotic contraction and application to matrix equations," Computational and Applied Mathematics, vol. 39, p. 301, 2020.

[25] H. Garai, L. K. Dey, W. Sintunavarat, S. Som, and S. Raha, "On new existence of a unique common solution to a pair of nonlinear matrix equations," 2020, https://arxiv.org/abs/2006. 10863. 